\title{
Accessibility and Utilisation of Antenatal Care (ANC) Services Among Pregnant Mothers Attending Antenatal Clinics. Case study: Mbale Regional Referral Hospital, Mbale, Uganda
}

\author{
Mayanja Jimmy ${ }^{1}$, Ilukor Geresom², \\ ${ }^{1}$ Mbale College of Health Sciences (Mbale School of Officers), Mbale, Uganda \\ ${ }^{2}$ School of Hygiene-Mbale, Mbale, Uganda \\ DOI: 10.29322/IJSRP.10.12.2020.p10805 \\ http://dx.doi.org/10.29322/IJSRP.10.12.2020.p10805
}

\section{Abstract}

Introduction: World Health Organization (WHO) passed a resolution that ANC is a basic human right and all pregnant women should receive irrespective of age, parity, race, faith of the pregnant women and many interventions have been put in place to ensure that worldwide and nationwide. ANC is a necessary component of maternal health in order to identify complications and danger signs during pregnancy. Despite the interventions that have been undertaken to truncate the maternal and infant mortality rates through ensuring improved access by women to reproductive health care services in Uganda; the effectiveness of the interventions has not been observed thus a study on accessibility and utilisation of ANC services was conducted at ANC clinic of MRRH, Mbale district.

Objectives: The general objective of the study was to establish the factors influencing the accessibility and utilization of ANC services among pregnant mothers attending ANC clinic in Mbale Regional Referral Hospital. Specific objectives were: to determine the level of utilization of ANC services among pregnant mothers attending ANC clinic in MRRH, to assess the quality of ANC services provided to pregnant mothers attending ANC clinic in MRRH and to establish mother's attitudes and suggestions on improving ANC services in MRRH, Mbale district. Methodology: This was a descriptive cross-sectional study. Ninety-seven (97) questionnaires were self-administered to the mothers from the ANC clinic during their visit. While three (3) interviews were held with the health workers. Data were presented and analyzed in tables and figures, interpreted using frequencies, percentages. Results: Many of the mothers were between age of 20-24 43(44.3\%), cohabiting mothers were the majority to be interviewed 47(48\%). Those with gravid 5 were $5(5 \%), 43(44 \%)$ had attained secondary education, $40 \%$ were peasants, $47(48 \%)$ had attended 2 visits, $76(78 \%)$ were aware of the importance of ANC early in pregnancy, 68 (70\%) said opening days were convenient for them, 83(86\%) didn't incur costs while utilizing ANC services. 93(96\%) rated health workers as rude. Among the mothers interviewed 67(69\%) received health education, 92(95\%) received drugs for prevention of Malaria and Anaemia, 17(18\%) were dewormed, 41(42\%) underwent screening for hypertension, HIV infection, syphilis. Conclusions: Factors that were found to be associated with the utilization of ANC services included. Age, marital status, parity, gravidity, tribe, educational level, income level status of pregnant mothers, awareness about ANC services, distance, health facility inadequacy and ANC provider behaviors.

Recommendations: The researchers recommend the following; recruitment of more health workers, more emphasis on health education and extension of more ANC services to the remote areas by the Ministry of Health to increase the availability of the services and increase their awareness about ANC services. Mothers are recommended to seek services immediately they realize they are pregnant.

Key Words: Accessibility, Utilisation, Antenatal care, Pregnant Mothers, Mbale, Ilukor, Mayanja 


\subsection{Introduction and Background to the study.}

\subsection{Background of the study}

Antenatal care is a planned programme of medical care offered to pregnant women by a skilled birth attendant, from the time of conception to delivery, aimed at ensuring a safe and satisfying pregnancy and birth outcomes. (UCG 2016, interactive)

According to the United Nations Millennium Development Goals (2012), every year, at least half a million women and girls needlessly die as a result of complications during pregnancy, childbirth or the 6 weeks following delivery. Almost all (99\%) of these deaths occur in developing countries. Reproductive health is a priority program for all African countries, including Uganda, a developing country, where maternal mortality continues to be a major problem.

World Health Organization (2009) passed a resolution that antenatal care is a basic human right and all pregnant women should receive irrespective of age, parity, race, faith of the pregnant women and many interventions have been put in place to ensure that worldwide and nationwide. ANC is a necessary component of maternal health in order to identify complications and danger signs during pregnancy. Regular ANC visits can provide some benefits for the women such as a strong relationship between women and the health care provider that can result in reducing complications during pregnancy. Women in rural areas in Uganda tend to receive less ANC visits than urban women. Developing countries account for more than $99 \%$ of all maternal deaths; about a half occurring in sub Saharan Africa and a third in South Asia.

There is a slow progress towards achieving the fifth goal of the Millennium Developments Goals (MDG) of 2020 in most developing countries including Uganda. The fifth goal aims to reduce the maternal mortality rate through ensuring that women access good quality reproductive health care and effective interventions some of which include ANC (WHO, 2005). But with failure to achieve the goal the maternal mortality will be seen to continue rising.

According to $\mathrm{WHO}$ (2003), ANC plays an important role in ensuring a healthy mother during pregnancy and a healthy baby after delivery. It was estimated that $25 \%$ the maternal deaths were mainly due to five major causes: hemorrhage; followed by eclampsia, infection (sepsis), abortion complications and obstructed labor yet such problems can be prevented since ANC programs are designed to maximize good health outcomes like low maternal mortality rates and low neonatal mortality, low cases of postpartum anemia and delivery of children with appropriate birth weight. Other issues compounding maternal mortality are lack of knowledge and preparedness about reproductive health in the family, community and health providers. Also there are inadequate numbers of reproductive health specialists to manage complicated pregnancies and deliveries and there is inadequate quality and access to all levels of obstetric care and other reproductive health service. To prevent unwanted outcomes of pregnancy, antenatal care (ANC) is the most important method for detecting pregnancy problems in the early period.

ANC is a critical element for reducing maternal mortality, and for providing pregnant women with a broad range of health promotion and preventive health services. One of the most important functions of ANC is to offer health information and services that can significantly improve the health of women and their infants. ANC is also an opportunity to inform women about the danger signs and symptoms for which immediate assistance should be sought from a health care provider. Furthermore, in many developing countries a Traditional Births Attendant (TBA) is still the dominant person, especially in rural areas. In Uganda, TBAs are popular with pregnant women for a number of reasons; such as not being expensive, similar beliefs, tolerant and kind. In addition, some of the reasons for high antenatal visit drop-outs in Mbale Uganda were: women perceived the need for the midwife only if there was a problem; women were more comfortable with a TBA because she spent more quality time with them and used a barter system for payment; women feared they could not afford to pay 
the price of the health center midwives; and women didn't have confidence in the new village midwives because they were often young, unmarried, and inexperienced.

According to a World Health Organization (WHO, 2015) report, the Indonesia Maternal Mortality Rate (MMR) continues in the high range of 420/100,000 live births, while coverage of births assisted by skilled providers is still low. And analytical review of the recent World Health Statistics showed that ANC coverage, between 2006 and 2013, was indirectly correlated with MMR worldwide. This indicates that countries with low ANC coverage are the countries with very high MR. For instance, ANC coverage in United Arab Emirates was $100 \%$ with MMR of 8per 100,000 and Ukraine had 99\% ANC coverage and MMR of 23\%. By comparison, in the sub Saharan Africa with ANC coverage of 49\%, Ghana had ANC coverage of 96\% and MMR of 380/100,000. Chad had $43 \%$ ANC coverage with MMR of 98/100,000 Nigeria had ANC coverage of $61 \%$ and MMR of over 560/100,000. Nigerias MRR is clearly above the African and global average of 500 and 210 respectively. In Kenya the maternal mortality rate of 1,000 per 100,000. (WHO, 2011). The poor maternal health outcome in Nigeria could be as a result of poor ANC utilization but could also be as a result of poor quality care. Despite the association of maternal health with quality of care the ANC coverage may not provide information on the quality of care provided.

The numbers of ANC visits vary depending on the country. In Western Europe, North America and many other countries ANC includes 12-16 visits to health care services by the pregnant woman, as well as provider visits to her home. Even so, the WHO and the Uganda government recommended a minimum of four ANC visits for a woman with a normal pregnancy; first visit being at 12 weeks but not late than 16 weeks of gestation, second visit at 24 weeks to 28 weeks, third visit at 32 weeks and fourth at 36 weeks. A review of studies from various countries indicates that the ANC utilization rate is still low due to many factors that need to be examined such as socio-demographic features, knowledge of social support and ANC services. Maternal and child welfare is not only related to health services provided by government and private organizations, it is also related to women as mothers including their education, economic status, culture, environment, and professional development. For example, a study in Xien Khouang Province, Lao PDR showed that significant predictors of ANC utilization were level of education, income, knowledge, attitude, distance to service, availability of public transportation, cost of transportation, and cost of services. Inadequate access and under-utilization of modern health care services were major reasons for poor health in developing countries. To improve planning and provision of ANC in a specific setting it is important first to be able to characterize those women and their families not receiving adequate care. Few studies with that perspective have been conducted in developing countries including Uganda.

\subsection{Statement of the problem.}

In the MRRH hospital profile written in November 2015, it showed an annual attendance of ANC of 28,141 women and an annual delivery number of 1014. The trend of the deliveries in the health facility and attendance of ANC, shows a close relationship between the two evidenced by the decreasing trend of attendance of ANC in view of this the researchers sought to establish the causes responsible for the trend of ANC utilization in MRRH. Therefore, the task of the study was to determine the level of utilization of ANC services among pregnant mothers and to assess the quality of ANC services provided to pregnant mothers as well as establishing mother's attitudes and suggestions on improving ANC services.

Antenatal care service utilization in developed countries of better compared to that in developing countries. In Uganda $48 \%$ of pregnant mothers who made four or more antenatal visits during pregnancy for their most recent births, less than $40 \%$ were assisted by a skilled health provider. (WHO/UNICEF, 2003), factors that have led to low utilization of ANC services include; lack of awareness and inadequate health education about ANC services, inadequate health workers, unskilled health workers who don't abide by medical ethics, long distance from the health facilities, low male participation in ANC services and traditional beliefs. 
Low utilization of ANC services has resulted into complications to both mother and new born, for example mother to child transmission of HIV/AIDS and any other risk factors in pregnancy like anemia which results into increased maternal and child mortality and morbidity rates.

\subsection{Objectives of the study}

The study was guided by both general and specific objectives

\subsubsection{General Objective}

The study aimed to establish the factors influencing the accessibility and utilization of ANC services among pregnant mothers attending ANC clinic in Mbale Regional Referral Hospital so as to reduce on infant morbidity and mortality rates.

\subsubsection{Specific Objectives.}

The study was guided by the following specific objectives;

a. To determine the level utilization of ANC services among pregnant mothers attending ANC clinics in MRRH.

b. To assess the quality of ANC services provided to pregnant mothers attending ANC clinics in MRRH.

c. To establish mother's attitudes and suggestions on improving ANC services in MRRH.

\subsection{Research questions}

The study sought to answer the following research questions;

a. What is the level of utilization of ANC services among pregnant mothers attending ANC clinics in MRRH?

b. What is the quality of ANC services provided to pregnant mothers attending ANC clinics in MRRH?

c. What are the mother's attitudes and suggestions on improving ANC services in MRRH?

\subsection{Justification of the study}

Despite the Uganda government efforts through the ministry of health to provide and promote ANC services, effects of not or underutilizing these services such as maternal and infant morbidity and mortality rates are still high. Low utilization of antenatal care services is the major cause of high maternal and child mortality rates in Uganda. it's important that factors affecting the utilization of antenatal services should be identified first in the communities.

Over the world studies have been conducted by different individuals on the factors influencing the accessibility and utilization of antenatal services among pregnant mothers. However not much is known about antenatal care in MRRH so there is a need to design most appropriate interventions for most pregnant mothers to attend, get access to and utilize antenatal services. My study will establish whether ANC services are utilized and will thus provide information which may be used in future to make appropriate interventions to improve on the accessibility, utilisation of ANC services by mothers and quality of ANC services effectively and efficiently

\subsection{Significance of the study}

This study was intended to fill in gaps on the existing information about the utilization of antenatal services by adding on the growing body of information needed by mothers, health workers of MRRH, MOH by providing strategic information to complement facility-based ANC services.

The findings in this study helped in developing appropriate message and opportunity that may in future help pregnant mothers to get access to and utilize antenatal care services effectively

This study determined the number of women who attend and utilize ANC services, assess the quality of ANC services provided to pregnant mothers and establishment of mother's attitudes and suggestions on improving ANC services and resources. The results of the study will be used to make necessary interventions to reduce morbidity, mortality and complications during pregnancy and delivery 
Their perspectives provided vital information for policymakers, health workers, donors, and communities on how to prevent infant, maternal mortality and morbidity rates.

\subsection{Scope of the study}

This was categorized as content scope and time scope and geographical scope

\subsubsection{Content scope}

The study was about accessibility and utilisation of antenatal care services among pregnant mothers attending ANC clinic, the level of utilization of ANC services and the quality of ANC services provided to pregnant mothers as well as the mother's attitudes and suggestions on improving ANC services.

\subsubsection{Time scope}

The study covered a period of five months that is from January to May 2018.

\subsubsection{Geographical scope}

The study was conducted in Mbale Regional Referral Hospital in Mbale district

\subsubsection{Respondent scope}

The study was limited to the pregnant mothers attending ANC in MRRH

\subsection{Limitations to the study}

The researchers encountered the following limitations in the study;

First, some health workers were not willing to give their opinions. Here, the researchers probed the health workers and got their opinions on the study.

Secondly, Financial resources to support research were inadequate since the researchers had to meet all the costs in the study. However, the researchers did their best to minimize the costs of the study as much as possible and seek financial support from family and friends

Thirdly, there was limited time to conduct the research due to the busy schedule of school activities.

Lastly, the researchers were also limited by language barrier since mothers spoke different language some of which was completely different from what the researchers knew.

\subsection{Literature Review}

Antenatal care is observed to be a necessity for every woman and the newborn baby. The researchers reviewed literature related to; the level of utilization of antenatal services among pregnant mothers attending ANC, the quality of ANC services provided to pregnant mothers in MRRH and mother's attitudes and suggestions on improving ANC services in MRRH. This information was obtained from several publications including textbooks, Reports, Journals, and the Internet sources.

\subsection{Level of Utilization of ANC services among pregnant mothers attending ANC.}

The experience of being pregnant encompasses physiological, psychological, spiritual and socio- cultural dimensions (WHO, 2003). Antenatal care (ANC) services are essential for a healthy pregnancy and child delivery though some workers argue that this is still an on-going debate (Adjiwanou \& LeGrand, 2013). Conrad et al. (2012) argue that experience from countries which have achieved low maternal mortality suggests that access to good-quality maternity services is critical to the improvement of maternal health. ANC is an interactive process between the pregnant woman and the medical personnel such as doctors, nurses and midwives. Like any other social interaction, it is influenced by socio-cultural definitions and perceptions particularly on pregnancy itself in this case. These definitions, perceptions and beliefs depend on the cultural and value systems.

In 2007, the World Health Organisation reported that 1,500 women die every day from pregnancy and childbirth related complications the world over. In 2005 it was estimated that 536,000 maternal deaths occurred 
worldwide due to pregnancy related complications (WHO,2007). Though in 2012 a $47 \%$ decline in annual maternal deaths was reported (UNFPA/UNICEF/WHO/ World Bank, 2012), it was still noted that many countries, particularly in sub-Saharan Africa will fail to reach the Millennium Development Goal target of reducing maternal death by $75 \%$ from 1990 to 2015 . Developing countries account for $99 \%$ of all the deaths and most of these deaths are due to direct causes of pregnancy complications (WHO, 2014). In developing countries, complications of pregnancy and child birth are the leading causes of death and disability among women within the reproductive age group. 46

Countries with good indicators in maternal and infant mortality have pregnancy related complications identified and managed early, however according to UBOS the overall one-time antenatal attendance in Uganda was found at $94 \%$ with women in rural areas being twice less likely to attend ANC than the urban women. According to the report only $8 \%$ of rural women in Uganda received ANC from a doctor. Regionally Southwestern Women were more likely to receive skilled care (20\%), than Eastern women (3\%), while only $2 \%$ of the women in Karamoja were reported to seek the same. It was reported that women in Uganda tend to seek antenatal care very late-37\% attending for the first time at 6 months or more. Globally, developing countries still face a challenge of poorly implemented ANC programs with irregular clinical visits and long waiting times plus poor feedback to the women. (United Nations, 2012).

A study in Hadiya zone, Ethiopia found that majority of the mothers who attended ANC did not receive adequate number of visits and initiated the visits later than recommended by the World Health Organization. A similar study done in Nigerian teaching hospital found that Nigerian women tended to obtain care late in pregnancy, and about one third the care was inadequate with almost half (47 percent) of women attending the ANC clinic in the third trimester. The ANC Service utilization in Ethiopia was significantly influenced by maternal age, where mothers aged between 25 - 29 years were less likely to utilize ANC service than women who were 35 years and older. Positive husband attitude towards ANC was also significantly related to ANC service utilization. Mothers' level of education influenced the use of ANC for which Mothers with primary educational level were more likely to attend ANC than women who are unable to read and write. This study further revealed that availability of women's time is important as women spend more time on their multiple responsibilities for care of children, collecting water or fuel, cooking, cleaning, and trade than on their own health (USAID, 2010).

In Hadiya; Ethiopia; Family size was a strong determinant of ANC service utilization with greater household size limiting the use of ANC service. A study done by Simkhada B, et al. (2015) also included maternal education, husband's education, marital status, availability, cost, household income, women's employment, media exposure and having a history of obstetric complications. But not leaving out Cultural beliefs, Parity and ideas about pregnancy. Whilst women of higher parity tend to use antenatal care less. Another study done in Ibadan, Nigeria (owoyokun KE, 2010) revealed that Women who were Muslims or other religions were more than 2 times likely to attend ANC clinic than women who were Christians. The same study showed that Women who were 25 years and older utilized ANC more than women who were below 25 years of age which agrees with study made in Bangladesh. (Schmidt j-O and Wang H, 2012). A study done in rural Local Government Area in Ogun State, Nigeria, identified that women preferred TBAs for various reasons which included: cheap easily accessible culturally acceptable services and more compassionate care than orthodox health workers, and for some it was the only maternity they knew. However, some respondents acknowledged that complications could arise from TBA care. (UNFPA, 2011) 
In many countries, TBAs are an important source of social and cultural support to women during childbirth and due to economic constraints, and the difficulty in posting trained professionals to rural areas; many women continue to deliver with TBAs. A study done in rural Uganda (Ebuehi OM and Akintujoje IA, 2012) revealed ANC attendance being irregular with few women appreciating the fact that ANC attendance was to monitor both the growth of the baby and the health status of the woman. This study also identified Parity as significantly influencing ANC attendance, but level of education, religion and marital status did not. Several factors influenced Ugandan women ANC seeking behavior which included: perceived high cost of (ANC services, conducting a delivery and treatment), and perceived inadequacy of services provided by the formal health system. Another study in India economic disparity along with cultural belief and restrictions determined care seeking behavior and utilization of health care, resulting in slow decline of child mortality rate. (Cathy Tukei, 2015), The recent Uganda Maternal Health review revealed that access to the basic antenatal care services has significantly declined. The Ministry of Health, Uganda in adherence of WHO (2016) recommends a simplified antenatal care of four visits; First visit: occurring in the first trimester, between $(10-20)$ week of pregnancy, second visit: scheduled close to week $26(20-28)$ of pregnancy, third visit: occurring in or around week 32 (28 - 36) of pregnancy, and lastly Fourth visit (final visit): taking place between weeks 36 and 38 (>36) of pregnancy.

\subsection{Quality of ANC services being provided.}

Quality of ANC has been designated one of the four Pillars of Safe Motherhood along with clean and safe delivery, essential obstetric care and family planning which could contribute to reduction of neonatal mortality (Akter et.al 2016)47and maternal mortality (Carroli, et al 2011).Good quality maternal health services are those which among others, are readily accessible, are safe, effective, acceptable to potential users and are the antenatal visits at a health facility staffed by technically competent people, provide prompt comprehensive care and/or linkages to other reproductive health services provide continuity of care and where staff are helpful, respectful and non-judgmental (Ashish et.al, 2016).

Quality of ANC depends on how women attend, initiate antenatal visits at a health facility. According to UNICEF/WHO(2013), about $70 \%$ of women worldwide had at least one antenatal visit with a skilled provider during pregnancy. ANC coverage was extremely high in the industralised countries, with $98 \%$ of women having at least one visit. In the developing world antenatal care use was around $68 \%$.The region of the world with the lowest levels of use was South Asia, where only 54\% of pregnant women have at least one antenatal care visit. In the Middle East and North Africa, use of antenatal care was somewhat higher (65\%). In sub-Saharan Africa, about $68 \%$ of women report at least one antenatal visit. The levels in the remaining regions of the world range from $82 \%$ to $86 \%$.

Availability of resources plays a bigger role in provision of quality ANC services(Valera, 2014).47 However, despite the high ANC attendance in most developing countries, a major problem hindering quality of ANC is inadequate resources. In a qualitative study done in Zimbabwe, health workers during in depth interviews expressed concern over shortage of antenatal resources such as drugs, staff, electricity and telephone and debilitated condition of the facility. Another study to assess quality of ANC IN Tanzania revealed that the quality of ANC was affected by lack of skills by staff, shortage of drugs, and inadequate stationary. Supply shortages (lack of drugs, equipment, gloves and reagents for urine and VDRL),infrastructure problems, and human resources to provide antenatal services have been cited as the major shortfalls in providing quality ANC 
in developing countries .Furthermore in many facilities often the available supplies are inadequate and erratic (Chintu \& Susu 2012). This is not unexceptional in Uganda though no study has come out clearly.

Substandard care resulting from poor staff supervision, underpayment, overworking and inadequate staff training or lack of refresher courses to upgrade staff skills have been reported in some studies. A study in Masawingo Zimbabwe revealed substandard care during antenatal period which resulted from lack of skills contributed to significant proportion of maternal deaths. (Boller, 2013).

\subsection{Mother's attitudes and suggestions on improving ANC services.}

Improve routine screening for pregnancy induced hypertensive disorders, anemia and syphilis Rationale: Hypertensive disorders, anemia and syphilis are some of the leading maternal conditions associated with still births in developing countries (WHO 2016). Screening for these conditions during antenatal care therefore facilitated early identification and hence treatment of these complications in a bid to reduce the high macerated still birth rate of 8.9/1000 live births (Jan - Dec 2014, baseline data) in the SMGL supported facilities in the region. Hypertensive disorders were the third leading cause of maternal death in 2015/16 in Uganda. Although preeclampsia may not be absolutely preventable, deaths due to this condition can be prevented by routine screening, which involves blood pressure measurements and urine testing. These must be done for all pregnant mothers in pregnancy and during labor as guided by the Ministry of Health, whose improvement Objective is to increase to $80 \%$ the number of ANC mothers screened and managed for pregnancy

According to the delivery of improved services for health (DISH) project (2000), their clients recommended that those concerned should address the following, qualified staffs, accessibility, long waiting times, provider behaviour, drug availability, maternal education, husband education, lowering costs of ANC services, reducing long distances from the health facilities, house income, men involvement and media exposure.

Identify and register pregnant women in the community, Specific Problem being addressed, Change concept, Change ideas tested, Steps in introducing the change ideas. Evidence that the changes led to improvement Scale of implementation, No information on existing pregnancies in the community, Develop a system of identifying pregnant women in the community Register women of child bearing age and evaluate for signs and symptoms of pregnancy. VHTs records all household members in the village register. For every woman of child bearing age registered, the VHT asks women if they experienced the signs of pregnancy on his list. Women who missed the past two menstrual periods are sent to health facilities for a test using a referral. Evaluated with medium rank 80 villages Ask women about possible pregnancies in the community. During community meetings or interactions with community members, a VHT asks non-pregnant women about pregnant women in their neighborhood. The VHT is informed about the locations of the pregnant women and are visited, Evaluated with high rank 80 villages Identify unregistered pregnant women during ANC visits, (UNICEF 2013).

All pregnant women attending ANC at health facilities are asked if they are registered by their VHT. VHTs are informed by the health worker about the women who are not registered in their villages. The household number from the village register is written on the mothers ANC record by the VHT as proof of registration. A mother missing this number implies no registration (WHO, 2015). Mothers also suggest that in order to escalate the numbers of pregnant women utilisating ANC services, key influencers should be involved e.g. mothers in law, use pregnant women to link their peers to VHTs, elder women, Traditional Birth Attendants, VHT talks to influence mothers during a home visit. VHT explains the benefit of early ANC to influencers. Using the list of 
pregnancy signs, he asks elder persons or mothers in-law, he also asks if there are suspected pregnancies in the household. ( USAID, 2017).

Follow up pregnant women for ANC, locate pregnancies in the community Update and identify homes of pregnant women on the village map. Raise awareness on benefits of ANC and facility delivery and danger signs, Involve opinion leaders in low uptake communities to raise awareness about importance of ANC and facility deliveries, Conduct focused dialogue meetings with pregnant women and community members to find reasons for low uptake for services and address concerns (WHO, 2017) .

\subsection{Methodology}

\subsection{Study design}

This study was a descriptive cross-sectional design. This design was chosen for study because it would generate results in a relatively short time and may serve as a foundation for further studies about ANC service utilisation.

\subsection{The study area.}

The study was carried out in antenatal clinic of MRRH in Mbale district Eastern Uganda. MRRH is located in Mbale municipality. Mbale district is $224.2 \mathrm{~km}$ from Kampala the capital city of Uganda. The hospital services benefit the following districts; Tororo, Sironko, Pallisa, Kumi, Bududa, Budaka, Busia, Manafwa. MRRH is a teaching and practicing hospital for medical students. The hospital has got 6 major departments which include; gynecological and obstetric department, surgery, pediatrics, medicine and psychiatric department. Antenatal care was my study area, under gynecological and obstetrics department. It is headed by a senior nursing officer who is the in-charge.

Activities carried out in ANC clinic in MRRH include: Health education /health promotion, Counseling to alert mothers on danger signs and help mothers to plan for child birth, Assessment of mothers which includes history taking, physical examination and screening tests, and giving prophylaxis medication and immunization against tetanus.

\subsection{Study variables}

The study variables were; 
Table 3.1 Showing the study variables

Independent Variables

\section{Dependent Variables}

-Level of Quality of ANC Services

-Level of Utilization of ANC Services

-Attitudes of Mothers

\subsection{Study population}

The study consisted of all pregnant mothers that attended the antenatal clinic department in April 2018.

\subsubsection{Selection criteria}

The selection of participants in the study depended on some factors and these factors led to either inclusion or exclusion from the study.

\subsubsection{Inclusion criteria}

All pregnant mothers who attended MRRH antenatal clinic in the month of April 2018 and who accepted to be interviewed after consenting were recruited in the study until a projected sample size was achieved.

\subsubsection{Exclusion criteria}

Mothers with the following characteristics were excluded;

All the mothers who didn't consent to the study and those unwilling to participate were excluded All mothers who were not residents of Mbale district.

\subsection{Sample size determination}

About $25 \%$ of the mothers attending ANC clinic in MRRH have children aged 0 to 5 years. Due to limited resources and for convenience purposes, a sample size of 100 respondents was used. The sample size of this study was calculated using the following formula:

$\mathrm{n}=\mathrm{QR} / \mathrm{O}$ (Bourbon's formula)

Where $n=$ sample size

$\mathrm{Q}=$ number of days spent in data collection.

$$
\begin{aligned}
& \mathrm{R}=\text { maximum number of respondents interviewed per day. } \\
& \mathrm{O}=\text { maximum time taken while interviewing. } \\
& \mathrm{Q}=10
\end{aligned}
$$




$$
\mathrm{R}=10
$$

$\mathrm{O}=1$

Then $\mathrm{n}=10 \times 10 / 1$

$$
\mathrm{n}=100 \text { respondents }
$$

Table 3.2: showing distribution of participants

\begin{tabular}{|l|l|}
\hline Category of respondents & Number \\
\hline Mothers & 97 \\
Health worker & 3 \\
\hline Total & $\mathbf{1 0 0}$ \\
\hline
\end{tabular}

\subsection{Sampling techniques.}

The researcher used purposive sampling and simple random sampling.

\subsubsection{Purposive sampling}

Health workers were selected using purposive sampling techniques. Purposive sampling refers to selecting samples based on the characteristics of the population and objective of study.

\subsubsection{Systematic random sampling}

Systematic random sampling technique was used to select mothers. Systematic sampling is a method in which sample members from a larger population are selected according to a random starting point and a fixed periodic interval

\subsection{Sampling procedure}

\subsubsection{Purposive sampling}

Health workers were selected since they were aware of the study and had knowledge about it. Those who were interested to participate were included in the study maximum of 3 (three).

\subsubsection{Systematic random sampling}

Mothers were selected systematically as every 3rd pregnant mother was selected to be part of the study. 


\subsection{Data collection methods}

Questionnaire survey and interviewing methods were used in data collection.

\subsubsection{Questionnaire survey}

The researcher used self-administered questionnaires to collect data from respondents.

\subsubsection{Interviews}

In this method, the researchers met the respondents face to face and obtained information concerning the study under investigation. The interviews were conducted with an interview guide

\subsection{Data collection tools.}

\subsubsection{Questionnaire}

Ahuja (2005) defines a questionnaire as a form of interrelated questions prepared by the researcher based on objectives of the study. The data for the study was collected by use of a self-administered and structured questionnaire (see a copy in appendix IV). The questions gained insight on the factors influencing the utilization of ANC among pregnant mothers. The questionnaire was therefore used to collect quantitative data.

It was issued to mothers who visited the ANC clinic during the month of February 2018. After they had made informed consent by signing a consent form. For those who didn't know how to read or write but were willing to participate were assisted by the researcher.

\subsubsection{Interview guide}

Three interviews were held with health workers to get in depth information on the study especially the quality of services delivered and the health worker role in providing ANC services with the use of the interview guide (see a copy in appendix III).

\subsection{Data collection procedure}

After approval of the research proposal and data collection instruments by the Supervisor, an introductory letter was obtained from the Principal of Mbale School of Clinical Officers permitting the researchers to conduct research at ANC clinic in MRRH. This letter was given to the District Health Officer (DHO), Mbale district. The DHO sent the author to the in charge of MRRH who allowed the author to carry out the study in that area. The hospital staff informed the respondents, then the interviewers introduced themselves and explained the study to the respondents 
The respondents participated in the study by informed consent either verbally or by signature. Data was collected using pretested questionnaire and was either be sent administered for literature respondents or by interview for illiterate respondents. At least 10 respondents were interviewed each day for 10 days until 100 respondents were achieved.

\subsection{Data quality control}

Quality was ensured on data collection by allowing enough time for interviews, repeating some interviews with questionnaire which had incomplete work, explained the meaning of each question, translated questions to local languages and ensured confidentiality of the information that was collected

\subsection{Data analysis and presentation}

Data analysis was immediately done after data collection. Data was coded. The authors analyzed data using SPSS statistical package (version 20.0). After data analysis, data was presented in frequency tables and bar graphs. Descriptive statics such as mean, frequencies, and percentage were used.

\subsection{Ethical consideration.}

Ethical consideration in relation to scientific research is premised on the protection of the right of participants in such research projects.

The introduction letter (see a copy in appendix x) from the Academic Registrar, Mbale School of Clinical Officers was presented to the relevant authorities in MRRH who granted permission for the study to be conducted in the antenatal clinic. (see a copy in appendix ix)

The researchers assured the staff of MRRH that the data obtained was kept confidential.

The participants were duly informed that their participation in the research survey was purely voluntary. There was no punitive measure taken against those who declined to participate neither any reward for participation. The researcher explained in details the purpose of carrying out the study and highly safeguarded and observed the respondent's confidentiality and privacy by not writing or disclosing the respondent's name in the report.

All information therefore obtained in the course of the study was treated with confidentiality and was not used outside the scope of the study.

\subsection{Project Management and Administration.}

\subsubsection{Project administration}

The project was administered as follows; - 


\section{Table 3.3: Showing Project Administration}

\begin{tabular}{|l|l|}
\hline Title & Responsibility \\
\hline - Researcher & $\begin{array}{l}\text { Designing data collection tools, collecting and } \\
\text { analyzing data and report writing. }\end{array}$ \\
\hline - Research Assistant & - Collecting data \\
\hline - Data Entrant clerk & - Entering data into SPSS \\
\hline - Supervisor & - Reviewing the data and report \\
\hline
\end{tabular}

\subsubsection{Project Work Plan.}

This project took five months that is from January 2018 to May 2018 (see a copy of the work plan in appendix IV).

\subsubsection{Project Budget}

This project costed an estimated figure of seven hundred thousand shillings $(700,000)$. (see a copy in appendix $\mathrm{V}$ attached).

\subsection{Dissemination of results}

Five copies of this study were printed out and distributed as follows; One copy to the school administration, and the second copy to the administration of Mbale Regional Referral Hospital for reference purposes, the fourth copy was retained by the researchers for future references when doing research in a related field.

\subsection{Findings}

\subsection{Social-demographic characteristics}

This section presented the socio economic and demographic characteristics of respondents. The study considered the age, religion, tribe, marital status, number of lives births, level of education, participants' employment status.

Table 4.1.1 Showing the distribution of respondents by age.

\begin{tabular}{|l|l|l|}
\hline Age group (years) & Frequency $(\mathbf{n}=97)$ & Percentage $(\boldsymbol{\%})$ \\
\hline $15-19$ & 15 & 15.5 \\
$20-24$ & 43 & 44.3 \\
$25-29$ & 30 & 30.9 \\
\hline
\end{tabular}




\begin{tabular}{|l|l|l|}
\hline$>30$ & 09 & 9.3 \\
\hline Total & $\mathbf{9 7}$ & $\mathbf{1 0 0}$ \\
\hline
\end{tabular}

Source; Field data, (2018)

As seen in table 4.1.1 above, of the total 97 pregnant mothers recruited in the study, there were 15(15.5\%) were between the age of 15-19 years, 43(44.3\%) were between 20-24years, 30(30.9\%) were between 25-29 years, $09(9.3 \%)$ were above 30 years.

\section{Table 4.1.2 Showing the distribution of respondents' religion}

\begin{tabular}{|l|l|l|}
\hline Religion & Frequency $(\mathbf{n}=\mathbf{9 7})$ & Percentage $\mathbf{~ \% ) ~}$ \\
\hline Catholic & 48 & 49.5 \\
Muslim & 05 & 5.1 \\
Protestant & 25 & 25.8 \\
Others & 19 & 19.6 \\
\hline Total & 97 & $\mathbf{1 0 0}$ \\
\hline
\end{tabular}

Source; Field data, (2018)

Data in respondent's religion shows that 48(49.5\%) respondents were Catholics, 05(5.1\%) were Muslims, $25(25.8 \%)$ were protestants, $19(19.6 \%)$ were from other religions

Figure 4.1.1; Distribution of respondents according to tribe

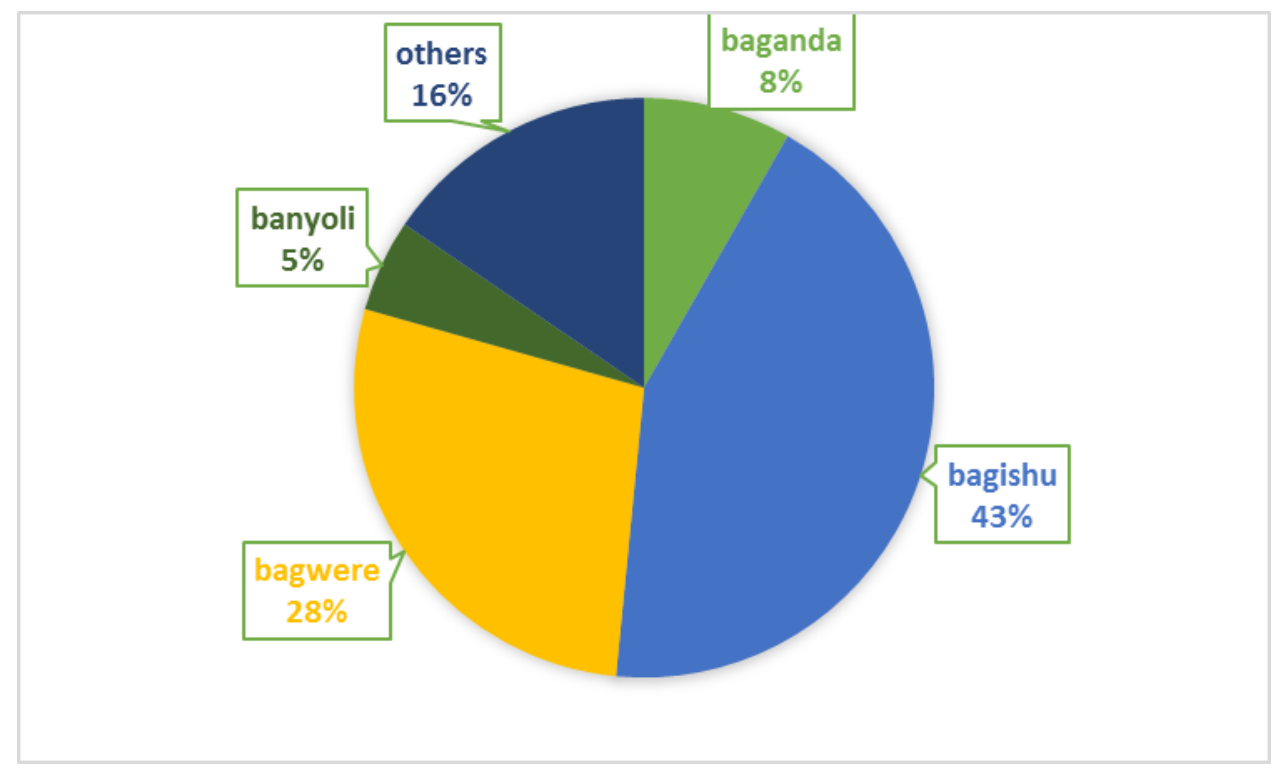

Source; Field data, (2018)

Majority of the respondents, 42(43\%) were Bagishu, 27(28\%) were Bagwere, 15(16\%) were from other languages ,8(8\%) were Baganda, and 5(5\%) were Banyoli. 


\section{Figure 4.1.2. Showing the distribution of respondents according to marital status}

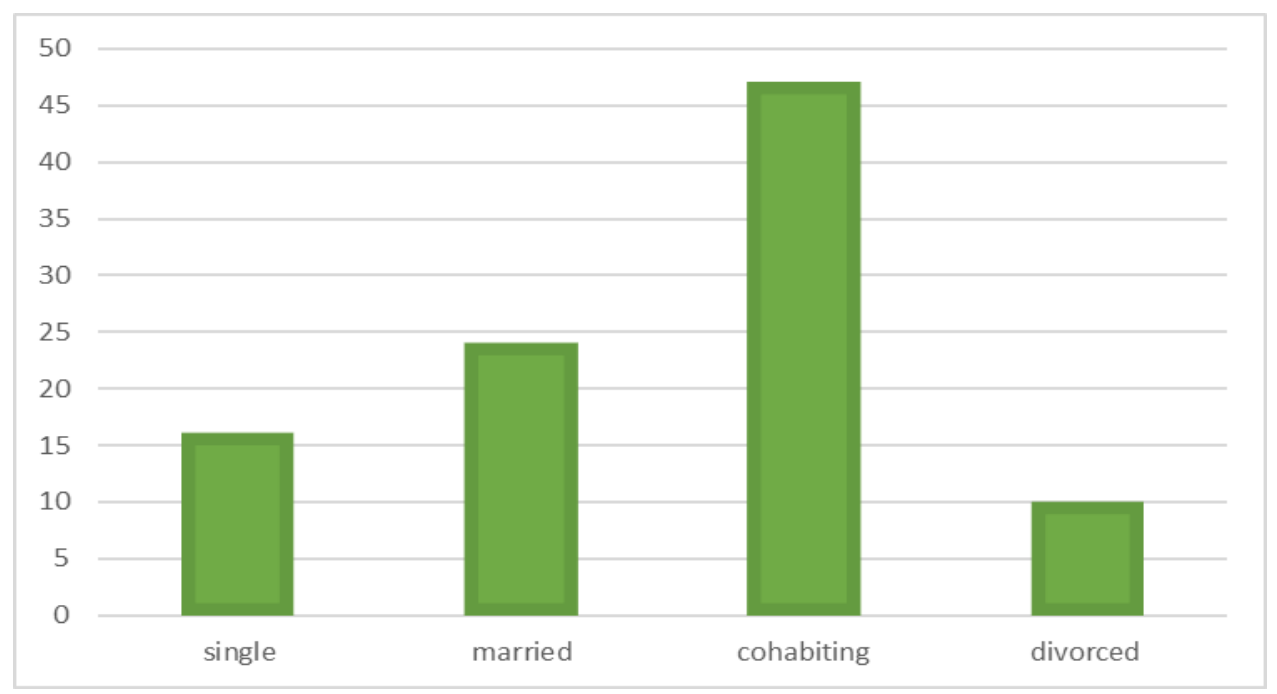

Source; Field data, (2018)

Of the pregnant mothers interviewed, 47(48\%) were cohabiting, 24(25\%) were married,16(17\%) were single. $10(10 \%)$ were divorced.

\section{Figure 4.1.3; Distribution of respondents by gravidity}

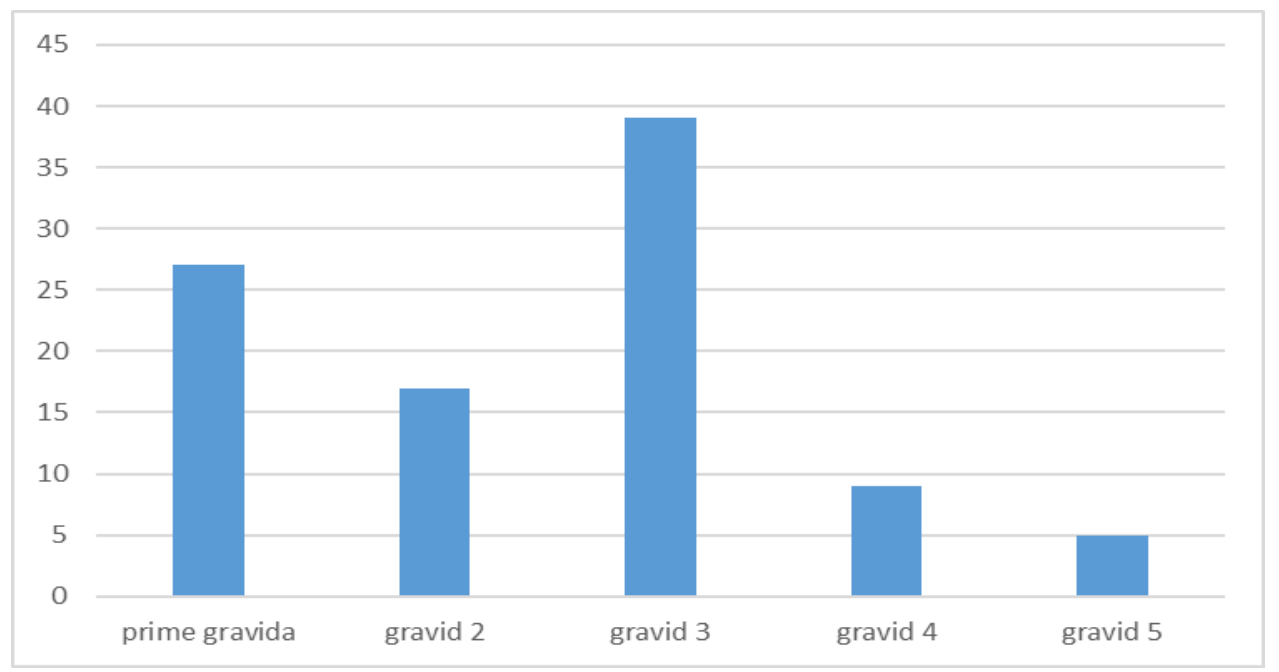

Source ; Field data, (2018)

More of the respondents were gravida $340 \%(39), 28 \%(27)$ prime gravida, $18 \%(17)$ gravida 2, 9(9\%) gravida 4 , and $5(5 \%)$ were gravida 5 . 
Figure 4.1.4: Distribution of respondents by the number of live births.

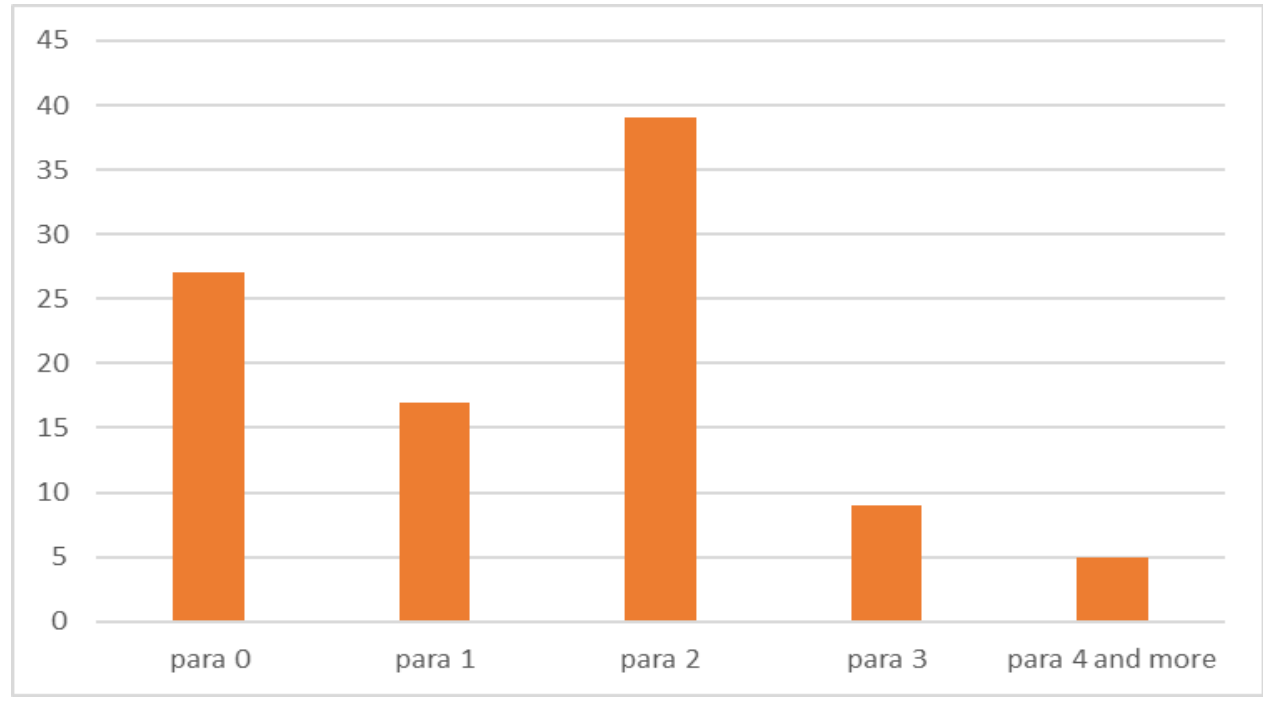

Source; Field data, (2018)

More of the respondents were para $240 \%(39), 28 \%(27)$ para $0,18 \%(17)$ para $1,9(9 \%)$ para 3, and 5(5\%) were para 4 and more.

Figure 4.1.5; Distribution of the gestation age of the pregnancy

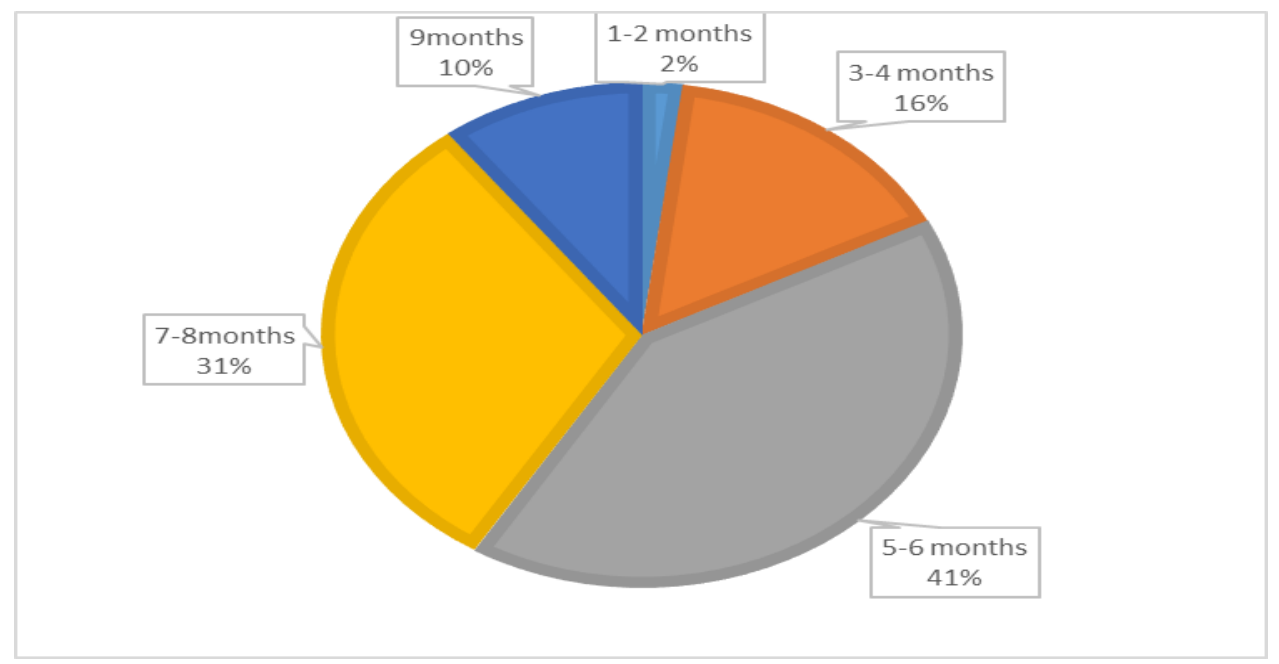

Source; Field data, (2018)

In relation with the gestation age of the pregnancy, more of the respondents were 5-6 months $40(41 \%), 30(31 \%)$ 7-8 months, $15(16 \%) 3-4$ months, $10(10 \%) 9$ months, and only $2(2 \%)$ were $1-2$ months. 
Figure 4.1. 6; Distribution of respondents according to level of education.

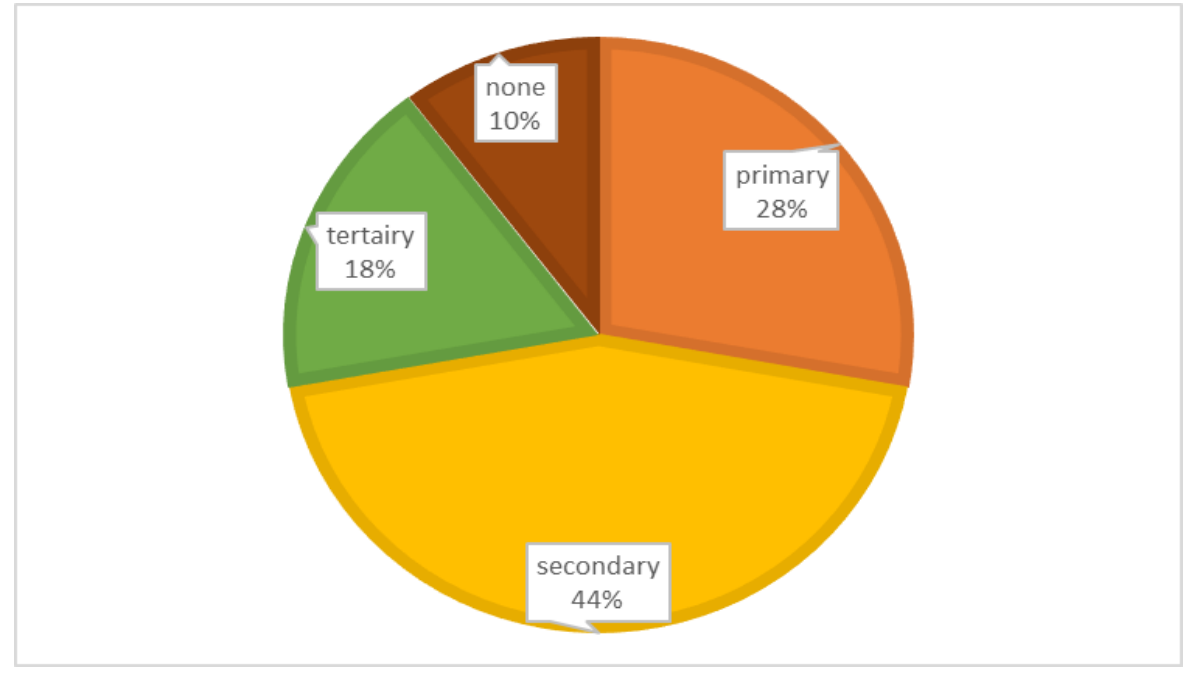

Source; Field data, (2018)

43(44\%) of the pregnant mothers attending ANC in MRRH had attained secondary education, 27 (28\%) had attained primary education,17 (18\%) had attained tertiary education and 10(10\%) had not attained any education..

Figure 4.1.7; Distribution of the respondents by occupation.

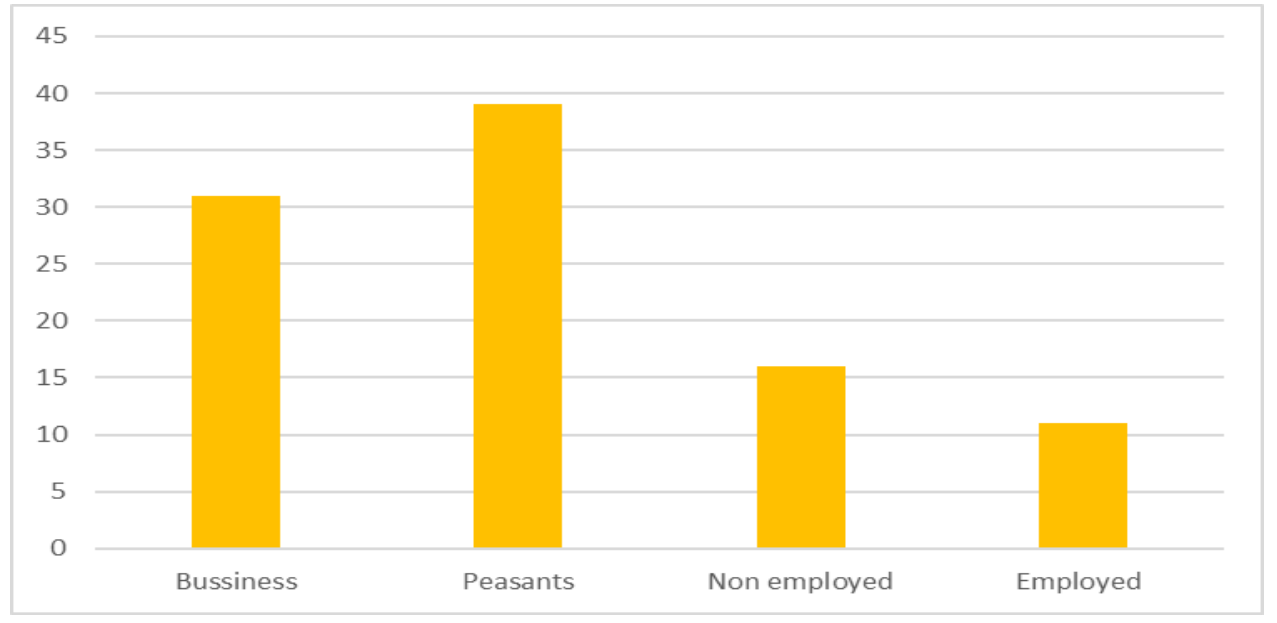

Source; Field data, (2018)

39(40\%) of the pregnant mothers attending ANC in MRRH were peasants, 31 (32\%) business, 16 (17\%) non employed, and 11(11\%) were employed. 
Figure 4.1.8; Distribution of the respondents by level of income.

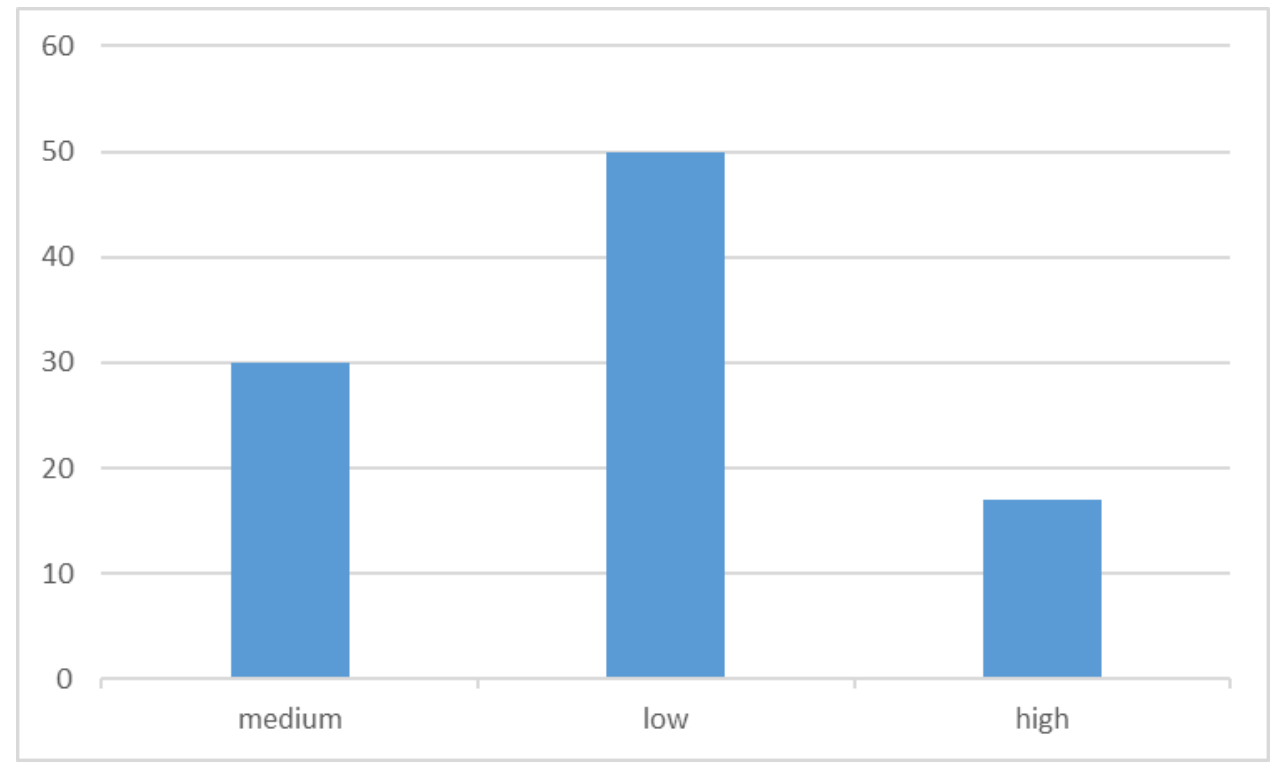

Source; Field data, (2018)

As indicated in figure 4.1.8, 50(52\%) of the respondents were low income earners, 30(31\%) medium, and $17(17 \%)$ were high earners. 
4.2 Objective one; To determine the level of utilization of ANC services among pregnant mothers attending ANC at MRRH.

This section presented the parameters to determine the level of utilization of the ANC services by the pregnant mothers.

Figure 4.2.1;.Showing the number of antenatal visits attended during present pregnancy

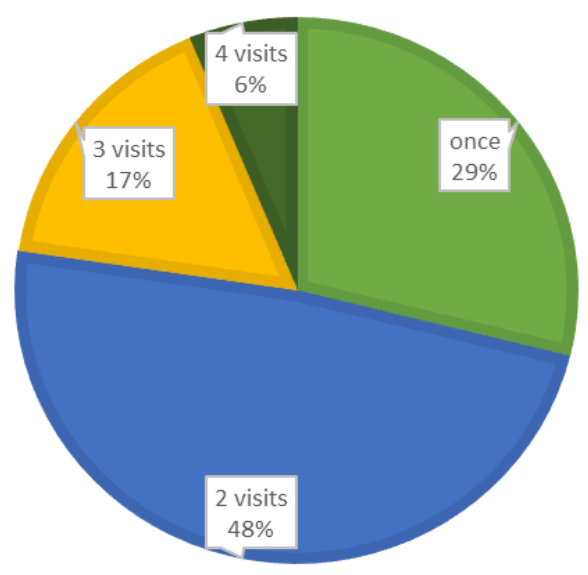

Source; Field data, (2018)

Among the respondents 47(48\%) were for their $2^{\text {nd }}$ visit, $28(29 \%)$ were for their $1^{\text {st }}$ visit, $16(17 \%)$ were for their $3^{\text {rd }}$ visit, $6(6 \%)$ were for their $4^{\text {th }}$ visit.

Figure 4.2.2; showing the distribution of respondents on whether they were using ANC services.

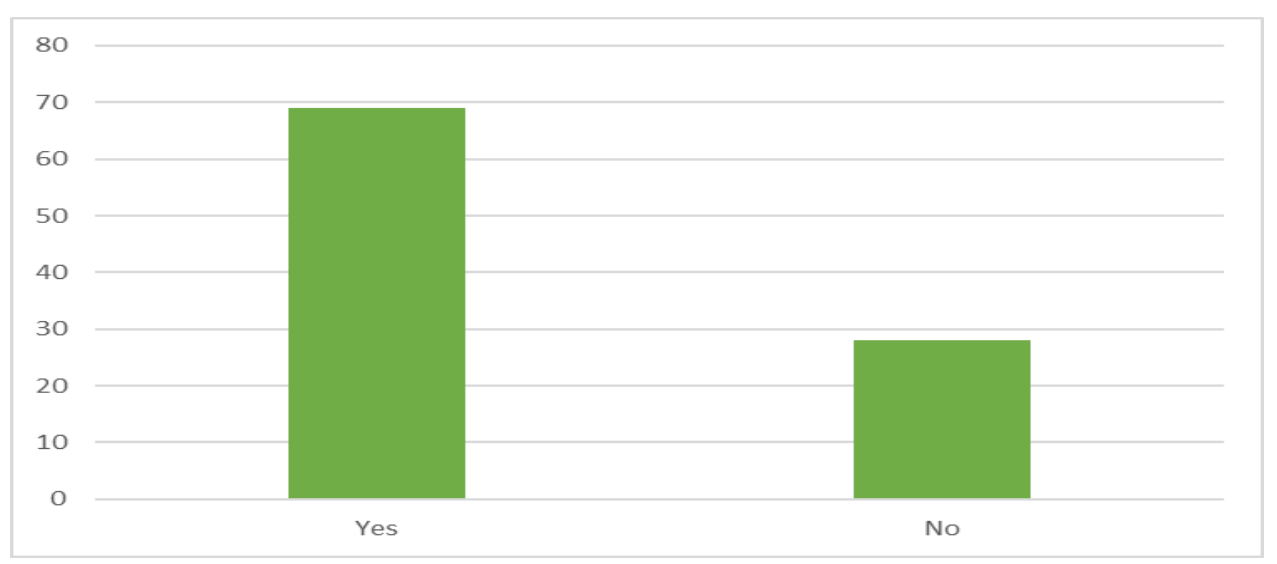

Source; Field data, (2018)

More than half of the respondents interviewed were using ANC services 69(71\%) and only 28(29\%) were not using ANC services. 
Figure 4.2.3; Showing the distribution on how far it was from respondent's home to the health facility.

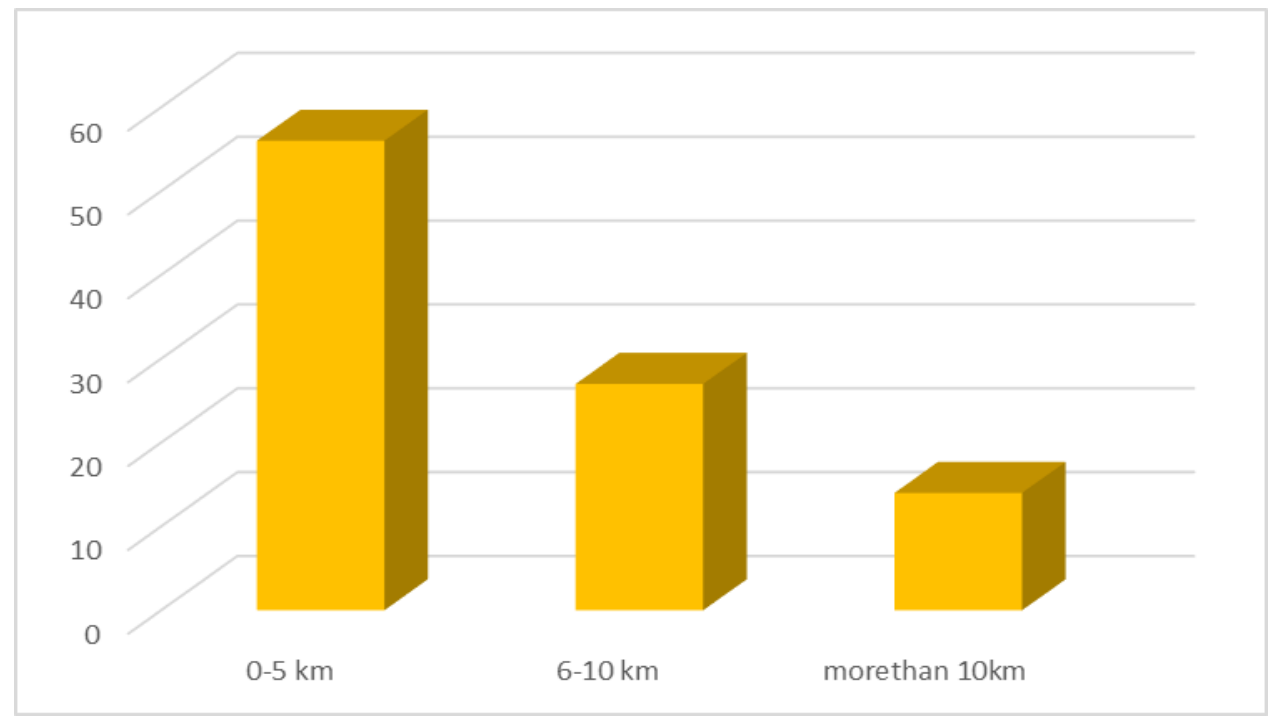

Source; Field data, (2018)

The majority of the respondents were 0-5 km from MRRH 56(58\%), 27(28\%) were 6-10 km, and 14(14\%) were more than $10 \mathrm{~km}$ away from MRRH.

Table 4.2.1; Showing distribution of respondents on their awareness about the opening days for ANC clinic.

\begin{tabular}{|l|l|l|}
\hline Days & Frequency(n) & Percentage (\%) \\
\hline Daily & 53 & 55 \\
Weekly & 8 & 8 \\
Twice a week & 7 & 7 \\
Monthly & 1 & 1 \\
Don't know & 28 & 29 \\
\hline Total & $\mathbf{9 7}$ & $\mathbf{1 0 0}$ \\
\hline
\end{tabular}

Source; Field data, (2018)

The biggest percentage of mothers 53(55\%) thought that ANC clinic was opened daily, 28(29\%) knew nothing, $8(8 \%)$ weekly, 7(7\%) twice a week, 1(1\%) monthly. 
Figure 4.2.5; showing distribution of respondents on whether the opening days of ANC clinic were convenient for them.

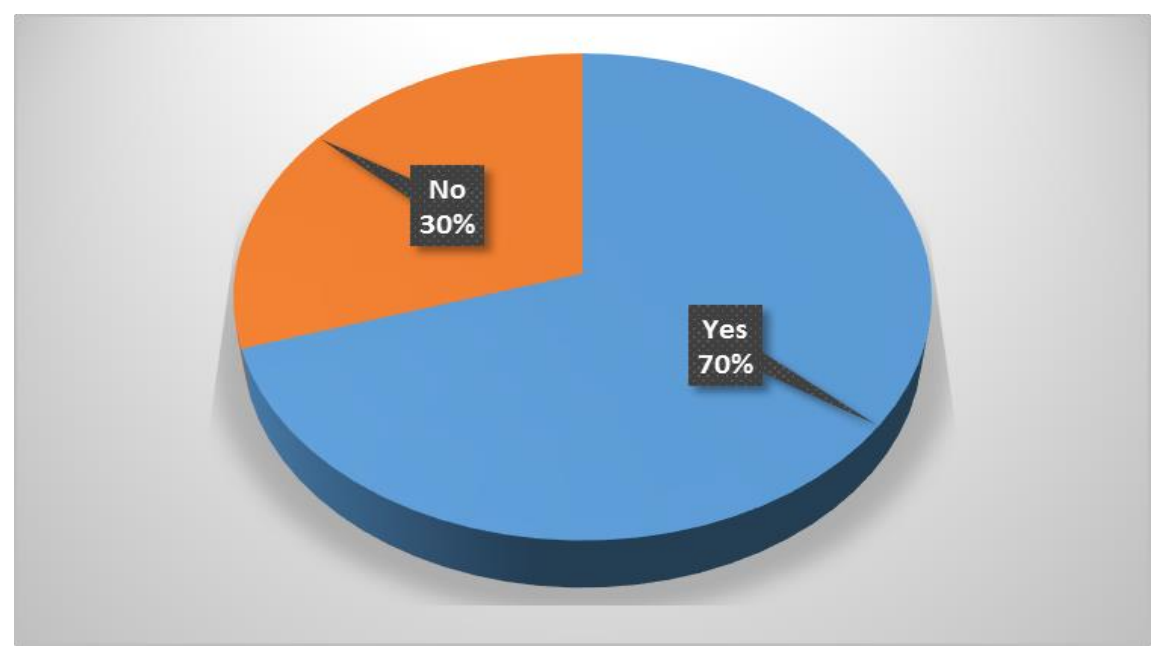

Source: Field data, (2018)

More than half of the pregnant mothers attending ANC in MRRH 68(70\%) said opening days were convenient for them and only $29(30 \%)$ said they were being inconvenienced by the opening days.

Figure 4.2.6; Showing the number of pregnant mothers who knew the importance of going for ANC early in pregnancy.

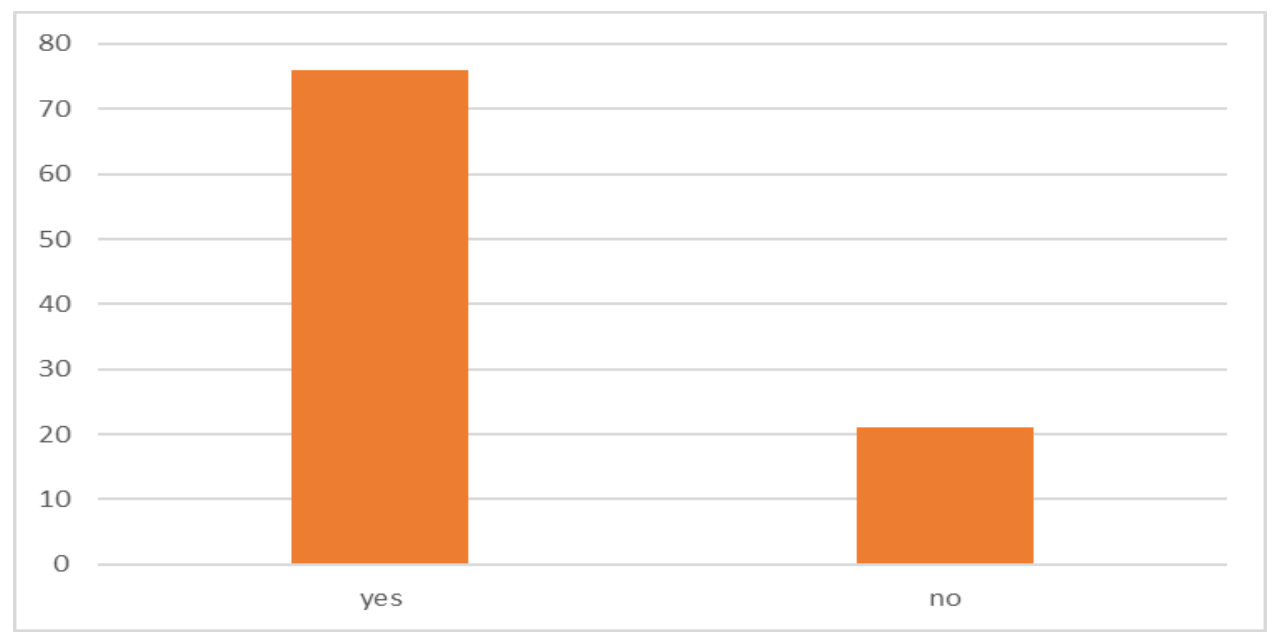

Source: Field data, (2018)

More than half of the pregnant mothers attending ANC in MRRH 76(78\%) were aware of the importance of attending ANC early in pregnancy and 21(22\% ) were not aware. 
Figure 4.2.4; Showing where respondents were planning to deliver from.

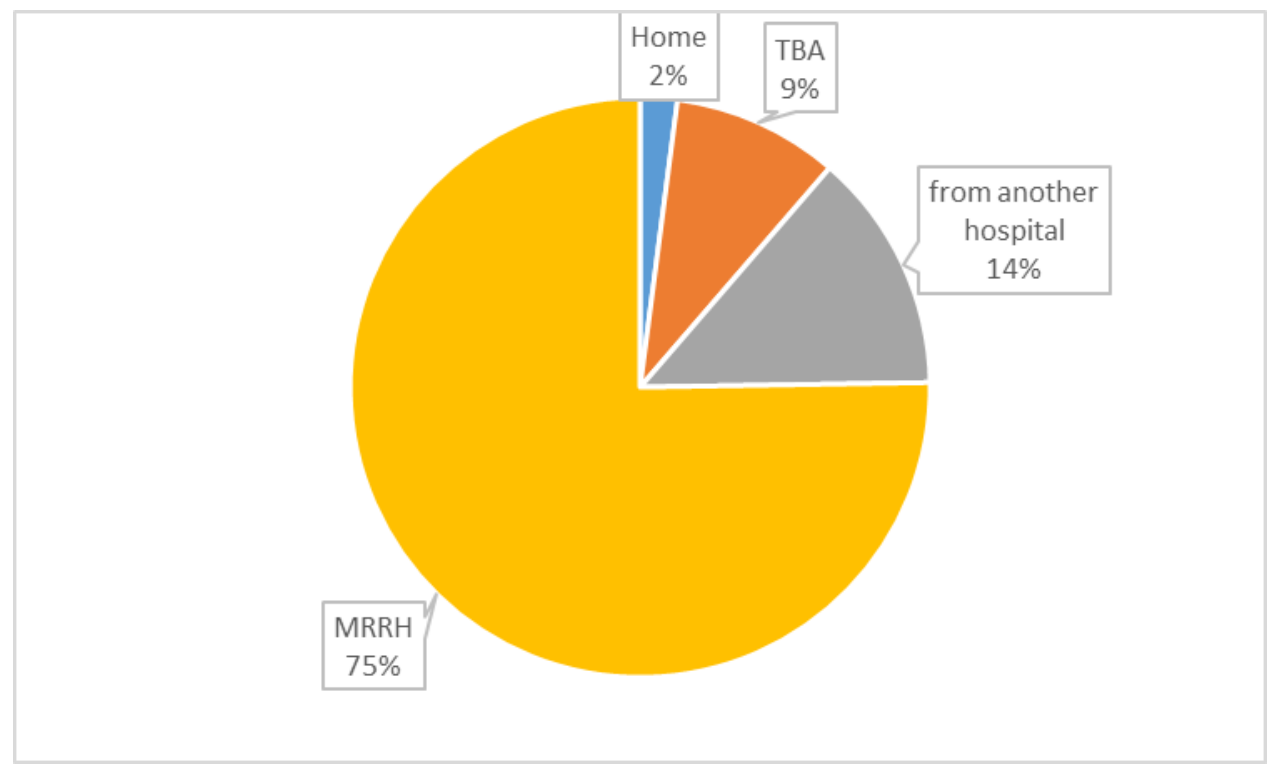

Source; Field data, (2018)

Majority of the pregnant mothers $73(75 \%)$ were planning to deliver from MRRH, 13(14\%) from another hospital, 9(9\%) from TBA, and 2(2\%) from home. 
4.3; Objective 2; To determine the quality of ANC services provided to pregnant mothers attending ANC clinic in MRRH.

This section presented the parameters used to determine the quality of ANC services provided to pregnant mothers attending ANC clinic in MRRH.

Figure 4.3.1: Showing distribution of respondents who incurred costs in utilizing ANC services.

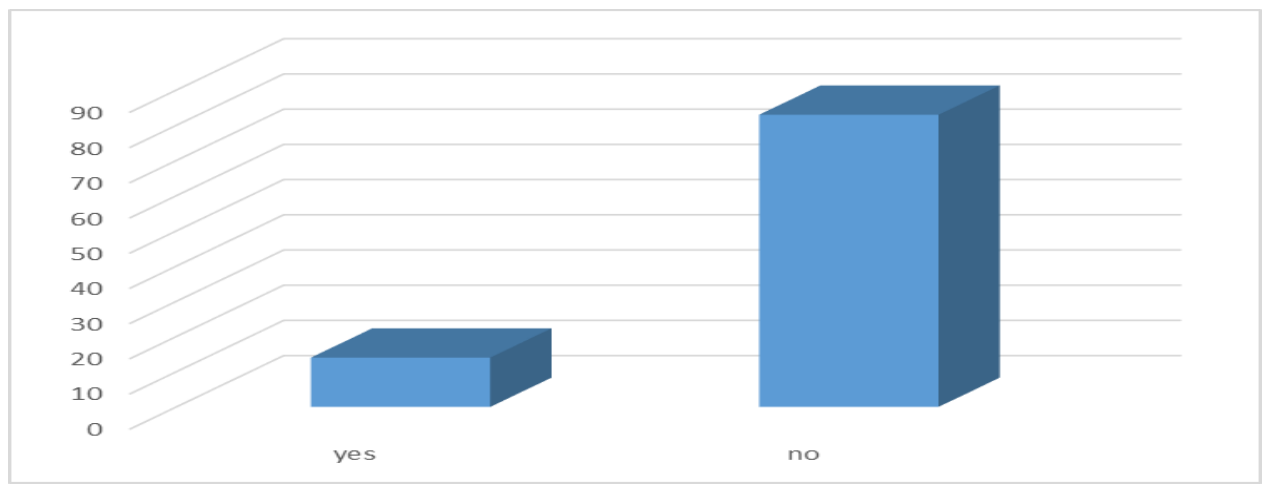

Source Field data, (2018)

Majority of the respondents 83(86\%) didn't incur costs while utilising ANC services and only 14(14\%) incurred costs during ANC.

Figure 4.3.2: Showing distribution of respondents that were hindered by the inadequate health facilities from accessing ANC on time.

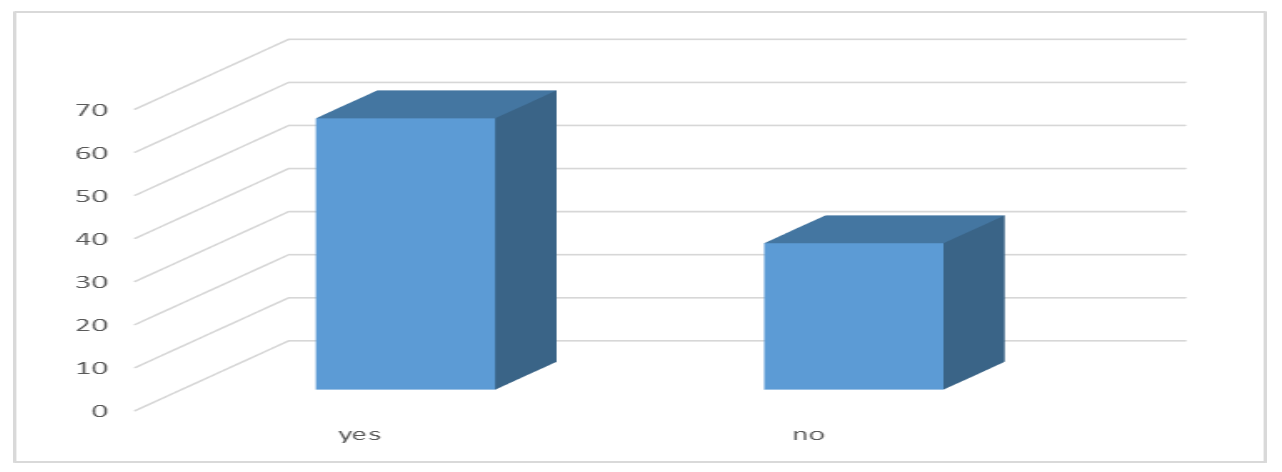

Source; Field data, (2018)

Majority of the respondents reported being affected by inadequate health facilities from accessing ANC on time and these added up to $63(65 \%)$ while only $34(35 \%)$ reported not being affected by the inadequate health facilities from accessing ANC on time. 
Figure 4.3.3; Showing distribution of respondent's rating of health workers.

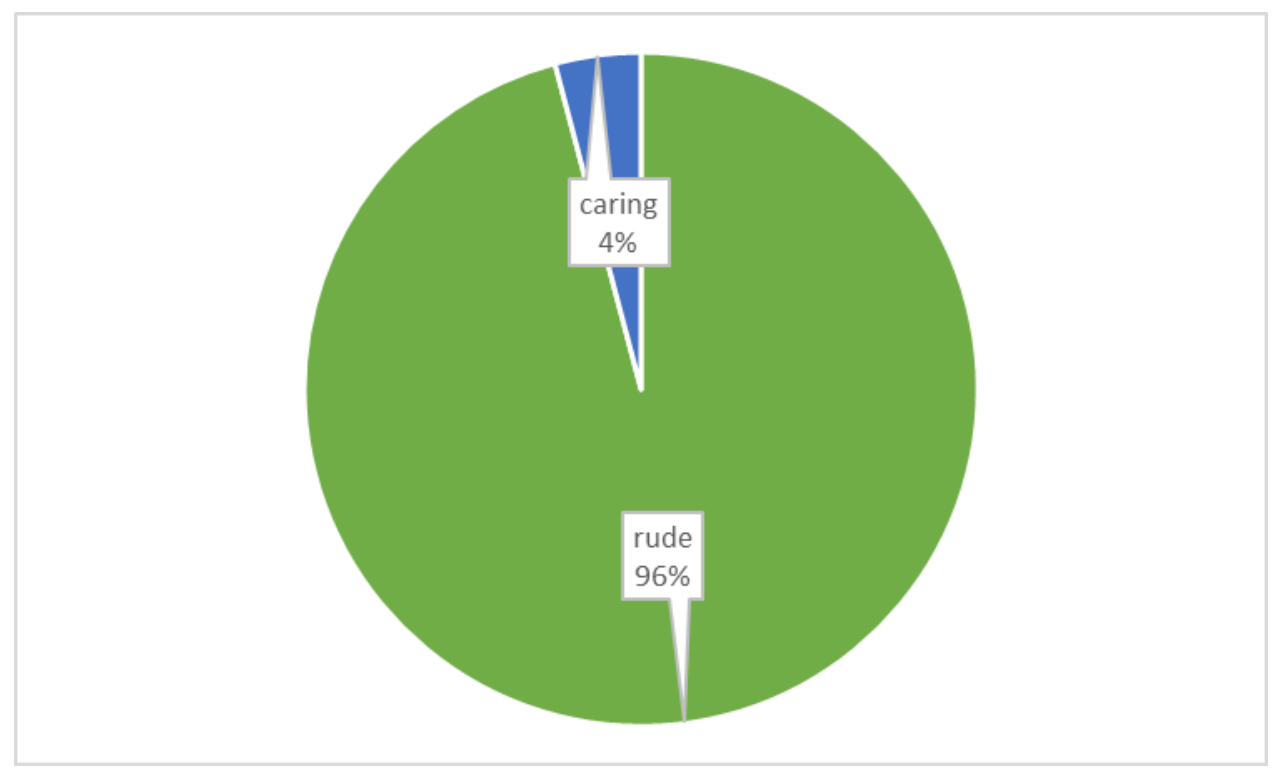

Source Field data, (2018)

Among the mothers interviewed majority 93(96\%) of them rated the health workers as rude and only 4(4\%) rated the health workers as caring.

Table 4.3.1; Showing distribution of ANC services were offered to pregnant mothers.

\begin{tabular}{|l|l|l|}
\hline ANC & Yes & No. \\
\hline Health Education & 67 & 30 \\
Drugs for prevention of malaria and anemia & 92 & 5 \\
Tetanus vaccination & 53 & 44 \\
Deworming & 17 & 80 \\
Examination of the pregnancy & 77 & 20 \\
Screening for hypertension, HIV infection, syphilis & 41 & 56 \\
\hline
\end{tabular}

Source; Field data, (2018)

Among the mothers interviewed 67(69\%) received a health education while 30(31\%) didn't, 92(95\%) received drugs for prevention of malaria and anemia while 5(5\%) didn't, 53(55\%) received Tetanus vaccine while 44(45\%) didn't, 17(18\%) were dewormed while 80(82\%) didn't, 77(79\%) underwent examination of the pregnancy while 20(21\%) didn't, 41(42\%) underwent screening for hypertension, HIV infection, syphilis while $56(58 \%)$ didn't. 
4.4; Objective 3; Mothers' attitudes and suggestions on improving ANC services.

\subsection{1; Qualitative data for pregnant mothers on the factors hindering ANC utilization and accessibility in}

\section{their area.}

On discussing with the mothers some of the things that hindered their accessibility to ANC services, uncertainty about when to start attending ANC services was one of them since mothers realize late that they pregnant. Mothers also reported shortage of ANC cards which leads to reprimands from health workers.

They also reported a difference in the attitudes and services provided by different health workers as some of them are rude, few are caring and few carryout the ANC procedures like checking fetal wellbeing. Some mothers reported language barrier as a hindering factor while others reported over waiting time.

\subsubsection{Qualitative data for pregnant mothers on their suggestions to improve ANC services in their area.} Mothers suggested that health workers should increase their awareness on when to start attending ANC clinic. Mothers also suggested that health workers should always provide ANC cards to avoid reprimands from health workers

They suggested that health workers should be empathetic while carrying out ANC services.

They also suggested that more health workers who are time conscious should be employed in order to reduce on waiting time.

\subsubsection{Qualitative data with the health workers}

When the health workers were interviewed out of the three interviewed one reported having done physical examination and laboratory investigations for every mother, they also reported opening for at least 6hours a day for the five working days of the week. They reported a low utilization on the ANC services which they attributed to the low level of awareness about the services since majority of the women usually started ANC by chance that is to say they come for ANC when they get a problem during the pregnancy.

\subsection{Discussions, Conclusions and Recommendations}

\subsection{Discussions}

\subsubsection{Social demographic data.}

The study shows that all the respondents 97 (100\%) were from 15years to above 30 years (Table 4.1) of the total 97 pregnant mothers recruited in the study, 15(15.5\%) were between the age of 15-19 years, 43(44.3\%) were between 20-24years, 30(30.9\%) were between 25-29 years, 09(9.3\%) were above 30 years. Due to the tender age, mothers between the age of 15-19years were afraid to seek health service care as they may be judged for being pregnant at an early age hence accounting for the low numbers attending ANC, thus agreeing with the study done in Ibadan, Nigeria (Owoyokun ME, 2010) where women aged 25 years and older utilized ANC more than those aged below 25 years which also agrees with the study made in Bangladesh.(Schmidt $j$-O 
and Wang H, 2012). Due to the preconception of experience in matters related to pregnancy and delivery accounts for the low numbers of mothers in the age range of 30 years and above. This is similar to the findings of the study done in Ghana by Christopher et al, (2015) where they found out that the youth (age group 24-29 years) frequently sought Antenatal care than any other age group.

Majority of the respondents 48(49.5\%) were Catholics, 05(5.1\%) were Muslims, 25(25.8\%) were protestants, 19(19.6\%) were from other religions. This study findings disagrees with the findings of study in Ibanda, Nigeria done by Owoyokun KE (2010) in which they revealed that Muslims or other religions were more than 2 times to attend ANC clinic than women who were christians.

Majority of the respondents 42(43\%) were Bagishu, 27(28\%) were Bagwere, 15(16\%) were from other languages ,8(8\%) were Baganda, and 5(5\%) were Banyoli. Most respondents were Bagishu because the study site was located in Bugishu land where Bagishu form the largest proportion of the population according to the year 2012 census.

Of the pregnant mothers interviewed 47(48\%) were cohabiting, 24(25\%) were married,16(17\%) were single. $10(10 \%)$ were divorced. The big number of married and cohabiting is attributed to the ignorance among women about family planning methods. Most of people in this area have false beliefs about family planning like becoming infertile after using contraceptives and Catholics being the biggest number and they are not recommended to use any of the family planning methods. This disagrees with a study done by Akter et. al (2016) where they said that inorder to reduce neonatal mortality, essential obstetric care and family planning should be emphasised through increasing mother's awareness about merits of family planning.

More of the respondents were gravida $340 \%$ (39),28\% (27) prime gravida, $18 \%$ (17) gravida 2, 9 (9\%) gravida 4, and $5(5 \%)$ were gravida 5 . This was associated to the fact that the greater the gravidity the greater the misconception of having enough experiences about issues concerning pregnancy and hence less attendance of ANC. This corresponds with the study done by Simkhada B, et al. (2015) in Hadiya, Ethiopia where they found out that women of higher gravidity tend to use antenatal care less.

More of the respondents were para $240 \%$ (39),28\%(27) para 0,18\%(17) para 1, 9(9\%) para 3, and 5(5\%) were para 4 and more. This was associated to the fact that the greater the parity, the greater the misconception of having enough experiences about issues concerning pregnancy and hence less attendance of ANC. This corresponds with the study done by Simkhada B, et al. (2015) in Hadiya, Ethiopia where they found out that women of higher parity tend to use antenatal care less. 
More of the respondents were 5-6 months 40(41\%), 30(31\%) 7-8 months, 15(16\%) 3-4 months, 10(10\%) 9 months, and only 2(2\%) were 1-2 months. Most of the respondents 40 (41\%) started attending ANC clinics at 56 months of pregnancy because this is the time when most women realize that they are pregnant and start looking for where to deliver from. This corresponds with a study carried out by Anuja et. al (2008) about utilisation of ANC in developing countries and United nations (2012) which indicated that most pregnant mothers attend first and more visits at 6 months or more.

As presented in the results, 43(44\%) of the pregnant mothers attending ANC in MRRH had attained secondary education, 27 (28\%) had attained primary education,17 (18\%) had attained tertiary education and 10(10\%) had not attained any education. This agrees with the study done by USAID (2010) in Ethiopia where mothers with primary educational level were more likely to attend ANC than women who are unable to read and write while a study in rural Uganda(Ebuehi OM and Akintujoje IA, 2012) revealed ANC attendance wasn't affected by level of education.

Majority of respondents 39(40\%) of the pregnant mothers attending ANC in MRRH were peasants, 31 (32\%) bussiness, $16(17 \%)$ non employed, and 11(11\%) were employed. This disagrees with the study done by Simkhada B, et al. (2015) were mothers with multiple responsibilities had less time for ANC services, lack of financial support for transport payments and private services accounted for low numbers of ANC attedance by unemployed mothers, this agrees with the findings by UNAIDS Inter-Agency Task Team on gender and also in line with Frontiers,(2015) findings in Eastern Nepal.

As indicated in figure 4.1.8 50(52\%) of the respondents were low income earners, 30(31\%) medium, and 17(17\%) were high earners. The low attendance numbers of higher earners was attributed to the multiple responsibilities limiting the time for pregnant mothers to attend ANC clinic thus agreeing with USAID(2010) study done in Hadiya zone.

\subsubsection{Objective One; To determine level of utilization of the ANC services among pregnant mothers attending ANC clinic in MRRH.}

The level of utilization of ANC services was determined by the following;

Among the respondents 47(48\%) were for their $2^{\text {nd }}$ visit, 28(29\%) were for their $1^{\text {st }}$ visit, $16(17 \%)$ were for their $3^{\text {rd }}$ visit, $6(6 \%)$ were for their $4^{\text {th }}$ visit. This shows there is an underutilization of ANC services since few of the pregnant mothers attended the four visits as recommended by WHO. This corresponds with the study done in Hadiya zone, Ethiopia by USAID (2010) where majority of the mothers who attended ANC didn't receive 
adequate number of visits which was attributed to negative husband attitude and lack of knowledge about the number of times, they have to attend ANC clinic.

More than half of the respondents interviewed were using ANC services 69(71\%) and only 28(29\%) were not using ANC services. The increased usage of ANC services is attributed to the improved service delivery thus disagreeing with the study done in rural local government Area in Ogun state, Nigeria, where women preferred TBAs for various reasons which included; cheap easily accessible culturally acceptable services and more compassionate care than orthodox health workers (UNFPA, 2011)

The majority of the respondents were 0-5 km from MRRH 56(58\%), 27(28\%) were 6-10 km, and 14(14\%) were more than 10km away from MRRH. 56(58\%) of the respondents lived within the vicinity of MRRH. This made it easier for most respondents to trend as many times as they required in order to utilize the ANC services hence agreeing with a study done by Valera (2014) that availability of ANC resources nearer plays a bigger role in ANC utilization.

The biggest percentage of mothers 53(55\%) thought that ANC clinic was opened daily, 28(29\%) knew nothing, $8(8 \%)$ weekly, $7(7 \%)$ twice a week, 1(1\%) monthly. The alarming false awareness about the opening days of ANC depicts low ANC attendance hence WHO (2017) encourages us to raise awareness on benefits of ANC utilisation and address concerns.

More than half of the pregnant mothers attending ANC in MRRH 68(70\%) said opening days were convenient for them and only 29(30\%) said they were being inconvenienced by the opening days. This depicts improved service delivery since the majority of the mothers are not affected by the opening days thus, corresponding with the study done in Tanzania by Chintu and Susu (2015).

More than half of the pregnant mothers attending ANC in MRRH 76(78\%) were aware of the importance of attending ANC early in pregnancy and 21(22\%) were not aware. Since the majority of the mothers in MRRH were aware of the importance of ANC attendance so my results disagreed with the study done in rural Uganda by Ebuehi OM and Akintujoje IA (2012) revealed ANC attendance being irregular with few women appreciating the fact that ANC attendance was to monitor both the growth of the baby and the health status of the woman.

Majority of the pregnant mothers 73(75\%) were planning to deliver from MRRH, 13(14\%) from another hospital, 9(9\%) from TBA, and 2(2\%) from home. The low number of planning deliveries at home and TBA`s is suggestive of the awareness on the importance of delivery from hospital to avoid risks and any other maternal 
complications which is corresponding to the study done by UNFPA (2011) in Ogun state, Nigeria where respondents acknowledged that complications could arise from TBA care.

\subsubsection{Objective Two; To assess the quality of ANC services provided to pregnant mothers attending ANC clinic in MRRH.}

The quality of ANC services was assessed using the following parameters;

Majority of the respondents 83(86\%) didn't incur costs while utilizing ANC services and only 14(14\%) incurred costs during ANC. Since the majority reported for not having incurred any costs, there is improved service delivery by health workers and hence utilization of the services thus matching with $W H O(2016)$ guideline recommendation which says all ANC services are free of charge. The low numbers of those who incurred costs corresponds with the study done in India by Cathy Tukei(2015) where economic disparity determined care seeking behaviour and utilisation of health care, resulting in slow decline of child mortality rates due to low ANC attendance which is meant to monitor fetal well-being(Akintujoje IA, 2012).

Majority of the respondents reported being affected by inadequate health facilities from accessing ANC on time and these added up to $63(65 \%)$ while only $34(35 \%)$ reported not being affected by the inadequate health facilities from accessing ANC on time. Adequate health facilities are readily accessible, are safe, effective, acceptable to potential users and ANC visits staffed by technically competent people, provide prompt comprehensive care and linkages to other reproductive health services provide continuity of care and where staff are helpful, respectful and non-judgmental(Ashish et. al, 2016). Inadequate services lead inconsistency in the services delivered, this agrees with the study done by Valera(2014) and Ebuehi OM (2012) in Uganda where inadequacy of services provided by formal health system affected ANC utilisation.

Among the mothers interviewed majority 93(96\%) of them rated the health workers as rude and only $4(4 \%)$ rated the health workers as caring. Rating the health workers as caring depicts a good mother- health worker relationship this increase mothers urge to seek for health services due to the friendly atmosphere and hence good compliance to the ANC services and therefore good utilization of the services (Christopher et al, 2013) hence attitudes of health workers doesn't affect the utilization of services in MRRH.

Among the mothers interviewed 67(69\%) received a Health education while 30(31\%) didn't, 92(95\%) received drugs for prevention of malaria and anemia while 5(5\%) didn't, 53(55\%) received Tetanus vaccine while 44(45\%) didn't, 17(18\%) were dewormed while 80(82\%) didn't, 77(79\%) underwent Examination of the pregnancy while $20(21 \%)$ didn't, 41(42\%) underwent screening for hypertension, HIV infection, syphilis while $56(58 \%)$ didn't. This was so because those services are free of charge in government health facilities. This 
corresponds with the WHO guidelines (2016) recommendations of ANC services, such as treatment of hypertension to prevent Eclampsia, preeclampsia, tetanus immunization, intermittent preventive treatment for malaria, elimination of mother to child transmission of HIV, micro nutrient supplement and information about danger signs during pregnancy and child birth.

\subsection{Conclusions.}

Based on the presentation, analysis and discussion of findings, the following conclusions were drawn chronologically as per the specific objectives.

\subsubsection{Social demographic factors.}

The social demographic factors found to affect the utilization of the ANC services were, extremes of age (either too young or old), marital status were the unmarried under-utilized the services, higher parity of the mother causing under-utilization, low education status leading to under-utilization, level of income status of mothers were by mothers with high income had the lowest utilization due to their busy schedule.

\subsubsection{Level of utilisation of ANC services.}

The level of utilization was found to be low since majority of mothers didn't attend 4 visits for ANC as recommended by WHO because of all the 97 respondents only $6(6 \%)$ were for their fourth visit.

\subsubsection{Quality of ANC services provided.}

Quality of the services was found to be moderately good since majority of the respondents didn't incur cost during utilization of the services, mothers were offered the following ANC services; Health education, deworming, screening for hypertension, HIV infection, syphilis, tetanus vaccination, drugs for prevention of malaria and anemia, examination of the pregnancy. Attitudes of health workers were rated rude by majority and health facilities inadequacy were found to affect the attendance.

\subsubsection{Mother's attitude towards ANC services.}

Mother's attitudes and suggestions on improving ANC services were increasing level of awareness among pregnant mothers about ANC by health workers since majority of the mothers were uncertain when to start ANC attendance, providing ANC cards to avoid reprimands from health workers, health workers should be empathetic while carrying out ANC services since majority of the respondents rated health workers as rude, more health workers who are time conscious should be employed to reduce on waiting time and more health facilities should be put in place to shorten distance thereby improving service delivery. 


\subsection{Recommendations.}

Since ANC directly affects the mortality rates of mothers and infants in Uganda and Mbale Regional Referral hospital in particular resulting into a nation burden. The researchers feel that the problem should be curbed only when efforts are combined with various bodies for example; Ministry of Health, Mbale Regional Referral Hospital, mother, and community members at large and therefore, the researchers came up with the following recommendations;

\subsubsection{Recommendations of Ministry of Health.}

- The Ministry of Health needs to put up interventions to increase the awareness about ANC services this can be through health talks on media like radio, TV, and these should be in the simplest language to be understood by majority and with all the necessary message they need to know.

- The Ministry of Health needs to put up more health facilities that can offer ANC services to mothers in the remote areas and also improve the already existing facilities in remote areas to reduce the work load in the referral hospital hence be able to use the available resources effectively.

\subsubsection{Recommendations to Mbale Regional Referral Hospital.}

- The management of Mbale Regional Referral hospital should recruit more health workers in the ANC department to avoid over working the available service providers and make the work quick hence short waiting time for the mothers

- The service providers of MRRH should be advised to put some more emphasis on the delivery of health education to mothers about ANC. Health workers should deliver message to mothers in the language they understand most and all health workers at large all those from others wards like medical or surgical should advise the mothers to go for ANC. MRRH staff should also continue sensitizing people through Health out reaches and on gatherings e.g schools, weddings, churches etc.

- Health workers in ANC clinic in Mbale Regional Regional Referral Hospital should be encouraged to regularly inform and explain to mothers about their findings from every check up and should be there to listen to any questions mothers may have as this may improve their understanding about ANC services provided to them and those they ought to receive.

\subsubsection{Recommendations to Mothers and Community members.}

- Mothers should be encouraged to always report to the hospital immediately they find out that they are pregnant and they should be encouraged to always keep the appointments for return dates they are given for subsequent visits. 


\subsection{Suggested area for further research.}

The researchers suggested the following area for further study;

- Factors influencing male participation in antenatal care in Mbale Regional Referral Hospital in Uganda.

\subsection{Acknowledgements}

The Researchers wish to acknowledge the different personalities and stakeholders that provided technical, moral and financial assistance during the study, most especially; the staff of Mbale regional referral hospital, Mbale College of Health Sciences, Mbale district health Office, and the participants who volunteered to take part in this study.

\subsection{Competing Interests}

None declared.

\subsection{References}

1. Abou Zahr.C, Wardlaw.T. (2004). Maternal mortality in 2000: estimates by WHO, UNICEF and UNFPA. Geneva: 2001.

2. Adekunle.C, V.Filippi, W.Graham, P.Onyemunwa, and E.Udjo.(1990). Patterns of maternity care among women in OndoStates, Nigeria. In Determinants of health and mortality in Africa, ed.AllanG.Hill, Demographic and Health Survey Further Analysis Series NewYork: The Population Council.

3. Bongiovanni.A, (1996): Health Project IV Workshop September 23-27; 1996: Jakarta, Indonesia. [Retrieved from; http://pdf.usaid.gov/pdf_docs/PNABZ677.pdf, accessed on $3^{\text {rd }}$ June 1998].

4. Desai.M, Phillips Howard.P.A, Odhiambo.F.O, Katana.A. Ouma.P.Hamel.M.J, (2013) Pregnancy related mortality in the KEMRI/CC health Demographic surveillance system in Western Kenya.

5. Dairo.M.D, Owoyokun.K.E, (2010). Factors Affecting the Utilization of Antenatal Care Services in Ibadan, Nigeria. Benin Journal of Postgraduate Medicine.

6. Ebuehi.O.M, Akintujoye.I.A, (2012). Perception and Utilization of Traditional Birth Attendants by Pregnant Women Attending Primary Health Care Clinics in a Rural Local Government Area in Ogun State, Nigeria. International Journal of Women's Health.

7. El-Sherbini.A.F, El-TorkyM.A, AshmawyA.A, Abdel-HamidH.S, (1993). Assessment of knowledge, attitudes and practices of expectant mothers in relation to antenatal care in Assiut governorate. J Egypt Public Health Assoc. 
8. Heidi.W.Reynolds, Emelita.L.Wong and Heidi Tucker, (2006). Adolescents' Use of Maternal and Child Health Services in Developing Countries International Family Planning Perspectives. Volume 32, Number1, March.

9. Kishk.N.A, (2002). Knowledge, attitudes and practices of women towards antenatal care: rural-urban comparison. J Egypt Public Health Assoc.

10. Karl.P, Ajegbomogun.B, (2005). Utilisation of Antenatal Care in a Nigerian Teaching Hospital. African Journal of Reproductive Health. 9:159-161. [PubMed]

11. Pembe.A.B, Carlstedt.A, Urassa.D.P, Lindmark.G, Nystrom.L, Darj.E, (2010). Quality antenatal care in Rural Tanzania: counseling on pregnancy danger signs. BMC Pregnancy and Childbirth, 10:35.

12. Maternal Mortality in 2005: Estimates Developed by WHO, UNICEF, UNFPA and the World bank. Geneva, Switzerland: World Health Organization, 2007

13. Ministry of Health: Indonesia Reproductive Health Profile The World Health Organization; 2003 [Retrieved from; http://www.searo.who.int/LinkFiles/Reporductive_Health_Profile_RHP-Indonesia.pdf, accessed on $19^{\mathrm{TH}}$ October 2004].

14. Ministry of Health: Indonesia Health Profile; 1997. [Retrieved from; http://http//www.depkes.go.id/ind/data/profile/index,htm, accessed on 25 th June 2000].

15. Mesganaw.F, Olwit.G, Shamebo.D, (1990). Determinants of ANC Attendance and Preference of Site or Delivery in Addis Ababa. Ethiopia Journal of Health Development.

16. Nguyen.H.T.H, Hatt.L, Islam.M, Sloan.N.L, Chowdhury.J, Schmidt.J-O, Hossain.A, Wang.H, (2012). Encouraging Maternal Health Service Utilization: An Evaluation of the Bangladesh Voucher Program. Social Science \& Medicine. 78:989-996. [PubMed]

17. Royston.E \& Armstrong.S, (1989). Preventing maternal deaths. The role of maternal health services. WHO Geneva: (pp153-183)

18. Saseendran Pallikadavath, Mary Foss.R.M and R.William Stones Antenatal Care in Rural Madhya Pradesh: Provision and Inequality. Population Resource Centre, Madhya.P.r

19. Safe Mother hood Initiatives, (2003). (Retrieved from: http://www.safe motherhood. org, accessed on 22 February 2004)

20. Simkhada.B, Teijlingen.E.R, Porter.M, Simkhada.P, (2008). Factors Affecting the Utilization of Antenatal Care in Developing Countries: Systematic Review of the Literature. Journal of Advanced Nursing. 61:244-260. [PubMed]. 
21. Titaley.R.C, Hunter.L.C, Dibley.J.M, Heywood.P, (2010): Why do some women prefer traditional birth attendants and home delivery?: A qualitative study on delivery care services in West Java Province, Indonesia. BMC Pregnancy and Childbirth, 10:43.

22. Thaddeys.S, Maine.D, (1994). Maternal mortality incontext SaiSai Med:38;1091-110.

23. UNFPA Indonesia, (2011). Reproductive and maternal health. [Retrieved from; http://indonesia.unfpa.org/mmr.htm, accessed on 2nd May 2014].

24. United Nations, (2009): The Millennium Development Goals Report. [Retrieved from; http://www.un.org/millennium goals, accessed on 4th October 2013].

25. Uganda Bureau of Statistics (UBOS) and Macro International Inc. Uganda Demographic and Health Survey 2006. Calverton: UBOS and Macro International Inc.; 2007.

26. USAID, (2007): Focused antenatal care: providing integrated, individualized care during pregnancy. [Retrieved from; http://www.accesstohealth.org/toolres/pdfs/ACCESStechbrief_FANC.pdf, accessed on 14th January 2008].

27. Villar.J, Bakketeig.L, Donner.A, et al, (2002). The WHO Antenatal Care Randomized Controlled Trial: Manual for Implementation of New Model. pp. 6-28.

28. WHO, UNICEF, and UNFPA. Maternal Mortality in 2000: Estimates Developed by WHO, UNCEF, and UNFPA. Geneva: 2003.

29. WHO \& UNICEF: Antenatal Care in Developing Countries: Promises, Achievement and Missed Opportunities: An Analysis of Trends, Levels, and Differentials. 1990-2001 Geneva: WHO \& UNICEF; 2003

30. WHO 2003: What is the efficacy/effectiveness of antenatal care and thefinancial and organizational $\begin{array}{llll}\text { implications? } & \text { (WHO } & \text { Europe). }\end{array}$ http://www.euro.who.int/_data/assets/pdf_file/0007/74662/E82996.pdf, accessed on 20th August 2005].

31. World Health Statistics(2008), [Retrieved from; http://www.who.int/whosis/whostat/2008/en/index.html, accessed on $5^{\text {th }}$ March 2012].

32. YaredMekonnen, AsnakechMekonnen, (2003). Factors influencing the use of maternal health care Services in Ethiopia. J Health Popul Nutr, Dec.

33. Ye.Y, Yoshida.Y, Rashid.O.R, Sakamoto.J, (2010). Factors affecting the utilization of antenatal care services among women in Kham District, Xieng KhouangProvince, Lao PDR. Nagoya J Med Sci, 72:2333.

34. YaredMekonnen, (2003). Patterns of maternity care service utilization in Southern Ethiopia: Evidence from a community and family survey. Ethiop.Health Dec. 
International Journal of Scientific and Research Publications, Volume 10, Issue 12, December 2020 ISSN 2250-3153

35. Zeine.A, Mirkuzie.W, Shimeles.O, (2010). Factors Influencing Antenatal Care Service Utilization in Hadiya Zone. Ethiopian Journal of Health Sciences. 20:75-82. [PMC free article] [PubMed]

36. World Bank. World Development Report: Infrastructure for Development. New York: Oxford University Press; 1994. 


\section{Appendices}

\section{Appendix I: Introductory Letter}

Telephones: 0785689788

0454437384

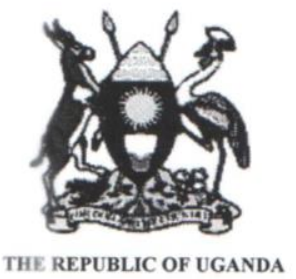

Ministry of Education \& Sports

Mbale School of Clinical Officers

P.O.BOX 1672

Mbale - Uganda

In any correspondence on this

Subject, please quote File No. MSOCO/047/INTL/018

The Hospital Director,

Mbale Regional Referral Hospital



$23 / 03 / 2018$

Mbale

\section{INTRODUCTION OF MAYANJA JIMMY}

Mayanja Jimmy Index number UA/DCM/056/15 is a third year student in Mbale School of Clinical Officers.

As a partial requirement for the fulfillment of the award of a Diploma in Clinical Medicine and Community Health (DCM), he is conducting a research titled "Factors influencing the accessibility and utilization of antenatal care services among pregnant mothers attending antenatal clinic in Mbale Regional Referral Hospital in Mbale district"

Any assistance accorded to him will be highly appreciated.

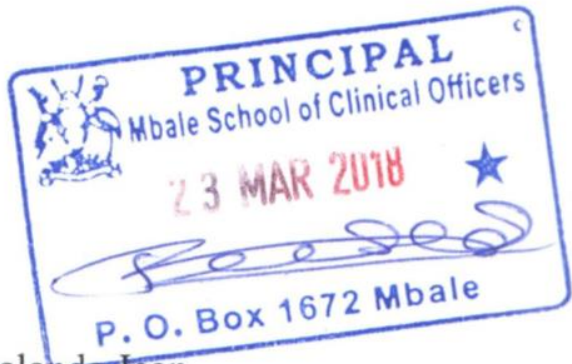

Babalanda Jean.

Principal 


\section{Appendix II: Letter of Aproval}

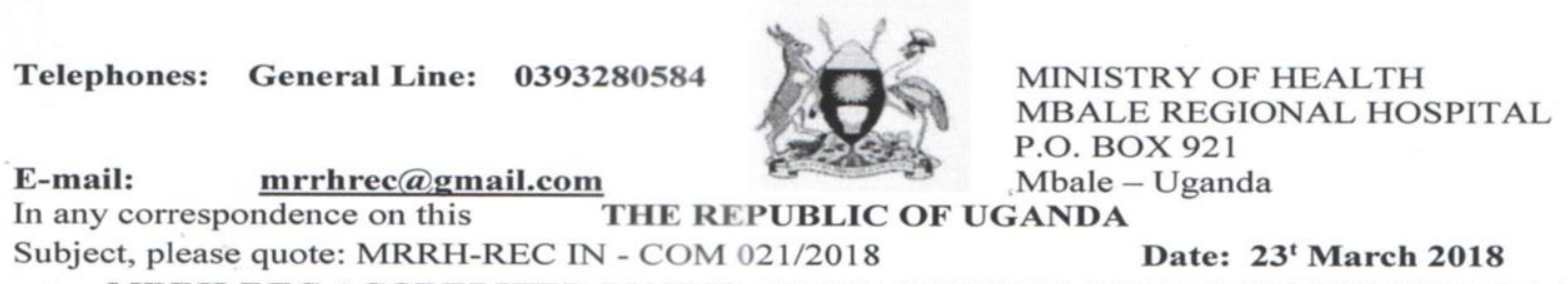

MRRH-REC ACCREDITED BY THE UNCST, REGISTRATION NUMBER UG-REC-011

Dear

MAYANJA JIMMY

UAHEB/DCM/056/15

Diploma student of Clinical Medicine

And community health SOCO

Principal Investigator

Study Title: FACTORS INFLUENCING THE ACCESSIBILITY AND UTILISATION OF ANTENATAL CARE SERVICES AMONG PREGNANT MOTHERS ATTENDING ANTENATAL CLINIC IN MBALE REGIONAL REFERRAL HOSPITAL

Ref: Full Ethical Approval for the study factors influencing the accessibility and utilization of antenatal care services among pregnant mothers attending antenatal clinic in Mbale regional referral hospital.

Thank you for submitting to Mbale Regional Referral Hospital-REC (MRRH-REC) the above study that we received on the $16^{\text {th }}$ March 2018 for research and ethical Approval. All the information submitted the committee was considered during the Administrative review, minute: 4 under SOP\#004 enacted on the 23rd March 2018.

\section{Confirmation of ethical opinion}

On behalf of the MRRH-REC, I am pleased to confirm a favorable opinion for the above research on the basis of submitted protocol and the additional supporting documents that are subject to the conditions below.

\section{Conditions of the favorable Ethical opinion}

Approval is contingent upon the received protocol and supporting documents submitted to MRRHREC on the $16^{\text {th }}$ March 2018 and the corresponding documents submitted on the same date. It is the responsibility of the principal investigator to ensure that protocol adherence is maximumly observed. In addition, the Principal investigator or their designee will be responsible for contacting the responsible Authorities in each of the study site(s).

MRRH-REC will be visiting your study site(s) to monitor protocol adherence. You shall submit to MRRH-REC a copy of your progress and final report(s). Consequently the submitted research proposal stipulated above is granted approval for implementation and will take immediate effect within the stamped date(s). If you wish to continue data collection or analysis beyond the approved date please submit an application for continuation approval at least two months before the expiry date.

In Addition to your application, the documents reviewed and approved in this meeting include:






\begin{tabular}{|c|c|c|c|}
\hline Document type & File name & Version & $\begin{array}{l}\text { Date of } \\
\text { submission }\end{array}$ \\
\hline \multicolumn{2}{|c|}{$\begin{array}{l}\text { Letter of introduction: from the principal investigator and } \\
\text { Mbale school of clinical officers. Dated } 23^{\text {rd }} \text { march } 2018\end{array}$} & NA & $23^{\text {rd }}$ March 2018 \\
\hline \multicolumn{2}{|c|}{$\begin{array}{l}\text { Study protocol } \\
\text { Factors influencing the accessibility and utilisation of } \\
\text { antenatal care services among pregnant mothers attending } \\
\text { antenatal clinic in Mbale regional referral hospital. }\end{array}$} & $\begin{array}{l}\text { March } \\
2018\end{array}$ & $16^{\text {th }}$ March 2018 \\
\hline \multicolumn{2}{|c|}{$\begin{array}{l}\text { Informed consent form } \\
\text { In English language appendix I page } 27\end{array}$} & $\begin{array}{l}\text { January } \\
2018\end{array}$ & $16^{\text {th }}$ March 2018 \\
\hline \multicolumn{2}{|c|}{$\begin{array}{l}\text { Data collection tools: use of questionnaire for direct } \\
\text { interview } 28-35 \text {, interview guide, page } 36\end{array}$} & $\begin{array}{l}\text { January } \\
2018\end{array}$ & $16^{\text {th }}$ March 2018 \\
\hline \multicolumn{2}{|c|}{$\begin{array}{l}\text { Time frame: four months as indicated by the dates of the } \\
\text { stamp. }\end{array}$} & NA & \\
\hline
\end{tabular}

On behalf of MRRH-REC, I wish you the best in your study.

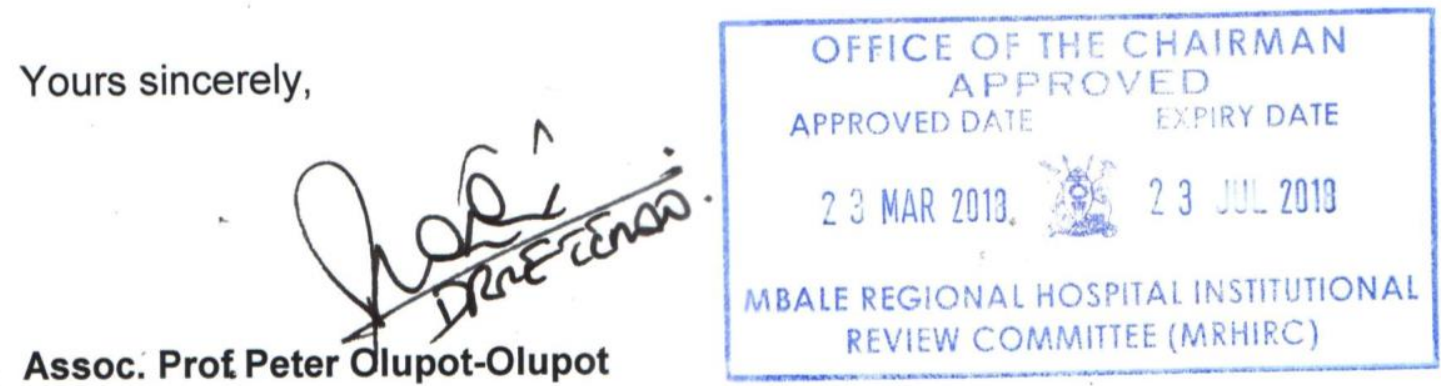

MB.Ch.B, MPH, PhD, Secretary General MRRH-REC 


\section{Appendix III: Consent Form}

\section{GOODMORNING / GOOD AFTERNOON MADAM/SIR,}

I am, Jimmy Mayanja, from Mbale School of Clinical Officers conducting a study on the factors influencing the utilization of ANC services among pregnant mothers attending ANC clinic in MRRH.

The purpose of the study is to identify the possible factors that affect the use of ANC services

The interview will approximately take 20 minutes and will be asked questions as in the questionnaire.

There are no monetary benefits for participating however by participating you will contribute to information that will assist Ministry of Health and policy makers to consider

community opinions as they formulate policies.

I would like to assure you that the personal information you disclose to me will not be disclosed to any third party unless required legally. Your identity will be kept anonymous by using a number to identify you instead of your name.

I am therefore requesting you to participate in this study. Your participation is solely voluntary and free to with draw from the study at anytime without penalty.

Your free to ask questions about this study.

Please sign this form if you have accepted to participate.

Thank you in advance,

Signature (thumbprint) of respondent.

Signature of Interviewee. 


\section{$\underline{\text { Topic }}$}

\section{Appendix IV: Questionnaire for Direct Interview}

Factors Affecting The Accessibility And Utilization Of Antenatal Care Services Among Pregnant Mothers Attending Anc Clinic In Mbale Regional Referral Hospital.

Date

Number

Am Mayanja Jimmy carrying out a "study to determine the factors affecting the accessibility and utilization of antenatal care services among pregnant mothers attending ANC clinic in MRRH".

Please participate in the study by answering the following questions as honestly as possible. No names are required and your responses will be treated with sincere confidentiality and will be used for the purpose of the study (TICK: the most appropriate answer where applicable.)

\section{SECTION A: SOCIO-DEMOGRAPHIC DATA}

1. How old are you?

$$
\begin{aligned}
& \text { 15-19 years } \\
& \text { 20-24 years } \\
& \text { 25-29 years } \\
& >30 \text { years }
\end{aligned}
$$

2. Religion.

Catholic

Muslim

Protestant

Others specify

3. Tribe
Mugishu
Muganda
Mugwere
Itesot
Munyoli

Others (specify)

4. What is your present marital status? 
Married

Divorced

Widowed

5. How many pregnancies have you had in total?

Number.

6. How many live births altogether?

1

2

3

4 or more

7. How old is your pregnancy?

1-2months

3-4months

5-6months

7-8months

9months

8. What is the highest educational level you attained?

Primary

Secondary

Tertiary

None

9. Occupation of the participant

Peasant

Business

Employed

Non employed 
10. Level of income

Medium

Low

High

\section{SECTION B: UTILIZATION OF ANTENATAL CARE SERVICES}

11. How many antenatal care visits have you attended during this pregnancy?



12. Have you been using ANC services?

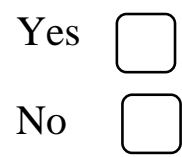

13. If no, what other alternatives have you been using?

14. How far is it from your home to this Health Facility?

$0-5 \mathrm{~km}$

$6-10 \mathrm{~km}$

More than $10 \mathrm{kms}$

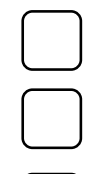

15. What mode of transport have you used to this health facility?

16. What are the opening days for ANC at this facility?

Daily

Weekly

Twice a week



don't know

Monthly

This publication is licensed under Creative Commons Attribution CC BY.

http://dx.doi.org/10.29322/IJSRP.10.12.2020.p10805 
17. Are the opening days convenient for you?

Yes



No

18. Give reasons for your answer above

19. Do you know why a pregnant woman should go for antenatal care early in pregnancy?

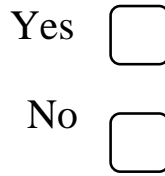

20. Give reasons why you go for antenatal care

21. Where are you planning to deliver from?

Home

TBA



From another hospital

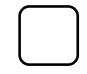

MRRH

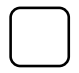

\section{SECTION C: QUALITY OF ANC SERVICES PROVIDED}

22. Do you incur any cost in utilizing ANC services?

Yes

No

23. Could inadequate health facilities prevent you from accessing ANC on time?

Yes

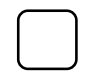

No

24. How do you rate the health workers in providing the services?

Rude

Caring

This publication is licensed under Creative Commons Attribution CC BY. 
25. Were you offered the following ANC services?

a. Health education.

Yes

No

b. Drugs for prevention of malaria and anemia.

Yes

No

c. Tetanus vaccination.

Yes

No

d. Deworming.

Yes $\square$

No

e. Examination of the pregnancy

Yes $\square$

No $\square$

f. Screening for hypertension, HIV infection, syphilis.

Yes $\square$

No

g. Any other services offered apart from the above, specify.

SECTION D: MOTHER'S ATTITUDES AND SUGGESTIONS ON IMPROVING ANC SERVICES.

26. What are the factors hindering ANC service utilization and accessibility in your area?

27. In your own view what can be done to improve on accessibility and utilization of ANC services in your area?

END

THANK YOU FOR YOUR COOPERATION, GOD BLESS YOU 


\section{Appendix V: Health Worker Interview Guide}

Factors Influencing The Utilisation of ANC Services Among Pregnant Mothers Attending ANC Clinic In MRRH

Date of Interview

Name of Facility.

\section{Demographic data}

1. Sex of Person being interviewed Female ( ) Male ( )

2. Age in years.

3. Level of education

4. Working experience

\section{Health workers role guiding questions}

1. What role do you play in the care of maternity client?....

2. What history do you take from antenatal mother who come to your clinic?

3. What do you check when doing physical examination?.

4. What Laboratory investigations do you carry out?

5. At the hospital, what time do you and close the ANC clinic?

6. What is you comment on the quality of antenatal services provided in the hospital?.

7. What is your comment on the women's utilization of the antenatal services? 


\section{Appendix VI: Workplan}

\begin{tabular}{|c|c|c|c|c|c|c|c|c|c|c|}
\hline & & & 2017 & & & 2018 & & & & \\
\hline $\mathbf{S N}$ & ACTIVITIES & PERSONNEL & OCT & NOV & DEC & JAN & FEB & MAR & APR & MAY \\
\hline 1 & $\begin{array}{l}\text { Proposal } \\
\text { development }\end{array}$ & $\begin{array}{l}\text { Researcher \& } \\
\text { Supervisor }\end{array}$ & & & & & & & & \\
\hline 2 & $\begin{array}{l}\text { Proposal } \\
\text { approval }\end{array}$ & Supervisor & & & & & & & & \\
\hline 3 & $\begin{array}{l}\text { Preparation of } \\
\text { data tools }\end{array}$ & Supervisor & & & & & & & & \\
\hline 4 & Pretesting tools & Researcher & & & & & & & & \\
\hline 5 & $\begin{array}{l}\text { Introductory } \\
\text { letter issuing }\end{array}$ & Supervisor & & & & & & & & \\
\hline 6 & $\begin{array}{l}\text { Permission from } \\
\text { local authority }\end{array}$ & Researcher & & & & & & & & \\
\hline 7 & $\begin{array}{l}\text { Data collection } \\
\text { process }\end{array}$ & Researcher & & & & & & & & \\
\hline 8 & $\begin{array}{l}\text { Analysis of the } \\
\text { findings }\end{array}$ & Researcher & & & & & & & & \\
\hline 9 & $\begin{array}{l}\text { Presentation of } \\
\text { findings }\end{array}$ & Researcher & & & & & & & & \\
\hline 10 & $\begin{array}{l}\text { Discussion and } \\
\text { conclusion }\end{array}$ & Researcher & & & & & & & & \\
\hline
\end{tabular}




\section{Appendix VII: The Research Project Budget}

\begin{tabular}{|c|c|c|c|c|c|}
\hline No. & Item & Description & Quantity & $\begin{array}{l}\text { Unit } \\
\text { cost }\end{array}$ & $\begin{array}{l}\text { Amount } \\
\text { (ugsh) }\end{array}$ \\
\hline 1 & \begin{tabular}{|l|} 
Stationary \\
\end{tabular} & \begin{aligned} \multicolumn{2}{l}{ This involves; } \\
$* \quad$ A ream of ruled papers \\
$* \quad$ A ream of photocopying papers \\
$* \quad$ Pens \\
$* \quad$ Long ruler \\
$* \quad$ Folder file \end{aligned} & $\begin{array}{l}1 \\
2 \\
5 \\
1 \\
2\end{array}$ & $\begin{array}{l}35000 \\
18000 \\
1000 \\
500 \\
6000\end{array}$ & $\begin{array}{l}35000 \\
\\
36,000 \\
5000 \\
500 \\
12,000\end{array}$ \\
\hline 2 & \begin{tabular}{|l|} 
Services \\
Printing
\end{tabular} & $\begin{array}{l}\text { This will be printing of the; } \\
\checkmark \text { Proposal } \\
\checkmark \text { Report }\end{array}$ & $\begin{array}{l}3 \\
3\end{array}$ & $\begin{array}{l}4,800 \\
9,000\end{array}$ & $\begin{array}{l}20,000 \\
30,000\end{array}$ \\
\hline & Photocopying & $\begin{array}{l}\text { This will be for; } \\
\checkmark\end{array}$ Questionnaires of four pages. & 150 & 800 & 120,000 \\
\hline & Binding & $\begin{array}{cc}\text { This will be for the; } \\
\checkmark & \text { Proposal copies } \\
\checkmark & \text { Report copies }\end{array}$ & $\begin{array}{l}3 \\
3\end{array}$ & $\begin{array}{l}3000 \\
3000\end{array}$ & $\begin{array}{l}9,000 \\
9,000\end{array}$ \\
\hline 3 & Transport & $\checkmark \quad$ Field transport & 16days & 2000 & 80,000 \\
\hline 4 & Lunch & $\checkmark \quad$ Balanced diet & 16days & 5000 & 100,000 \\
\hline 5 & Communication & $\checkmark \quad$ Airtime and internet & & 30,000 & 143,500 \\
\hline & Miscellaneous & & & 100,000 & 100,000 \\
\hline & Total & & & & $700,000 /=$ \\
\hline
\end{tabular}




\section{Appendix VIII: A Map of Uganda Showing Mbale District.}

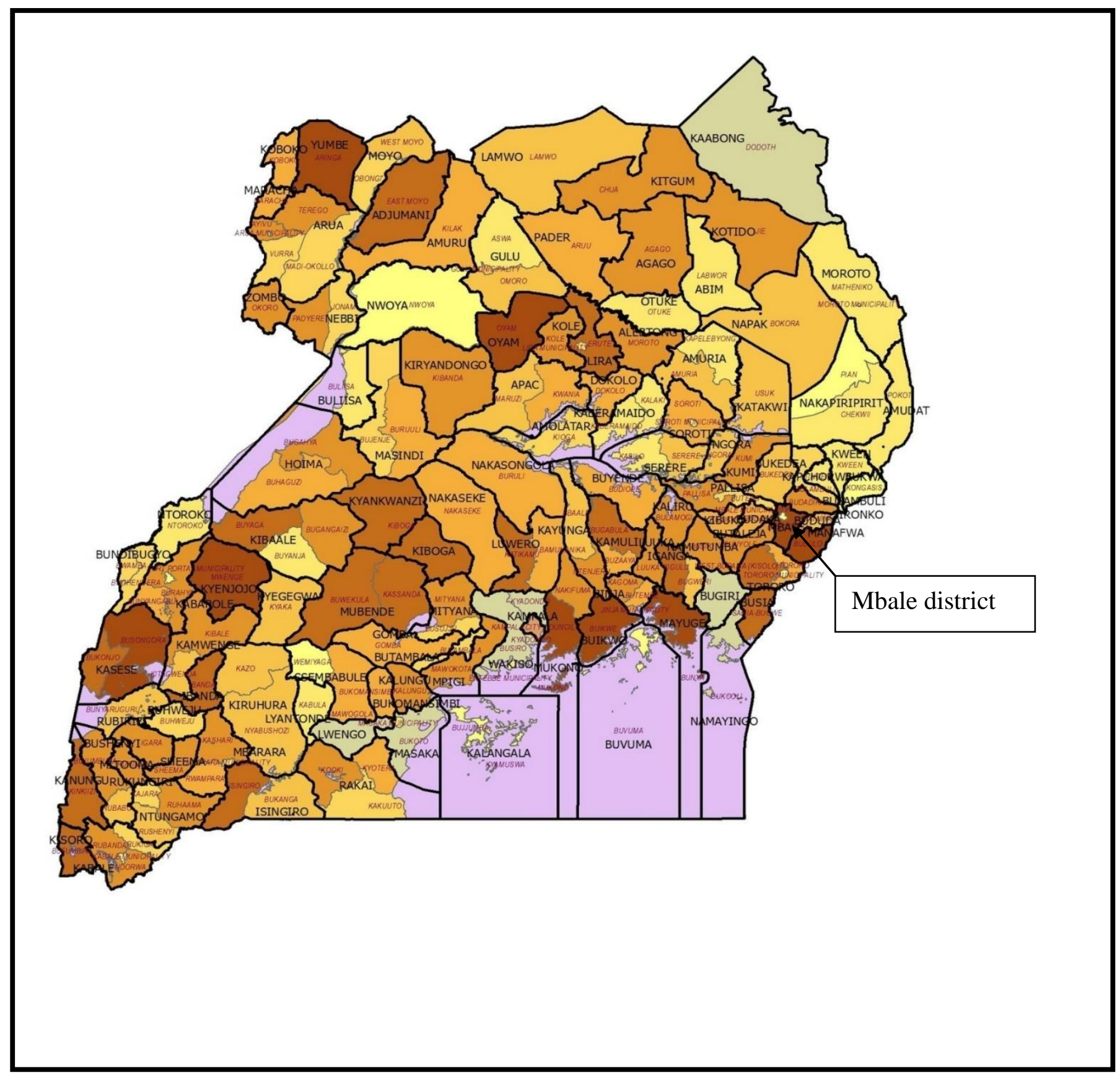


Appendix IX: A Map Of Mbale Showing The Study Area

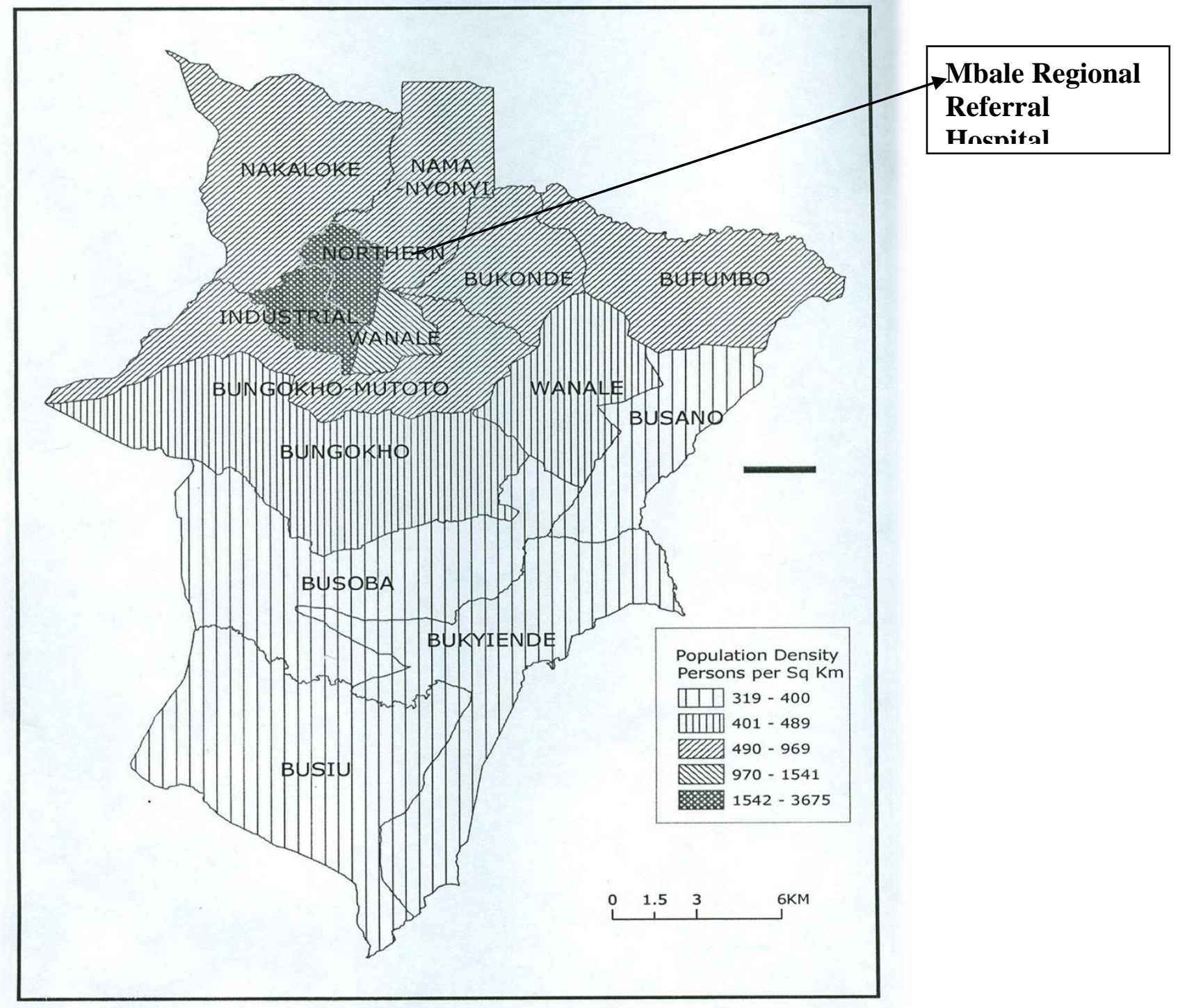




\section{Appendix X: Map of Mbale Regional Referral Hospital}

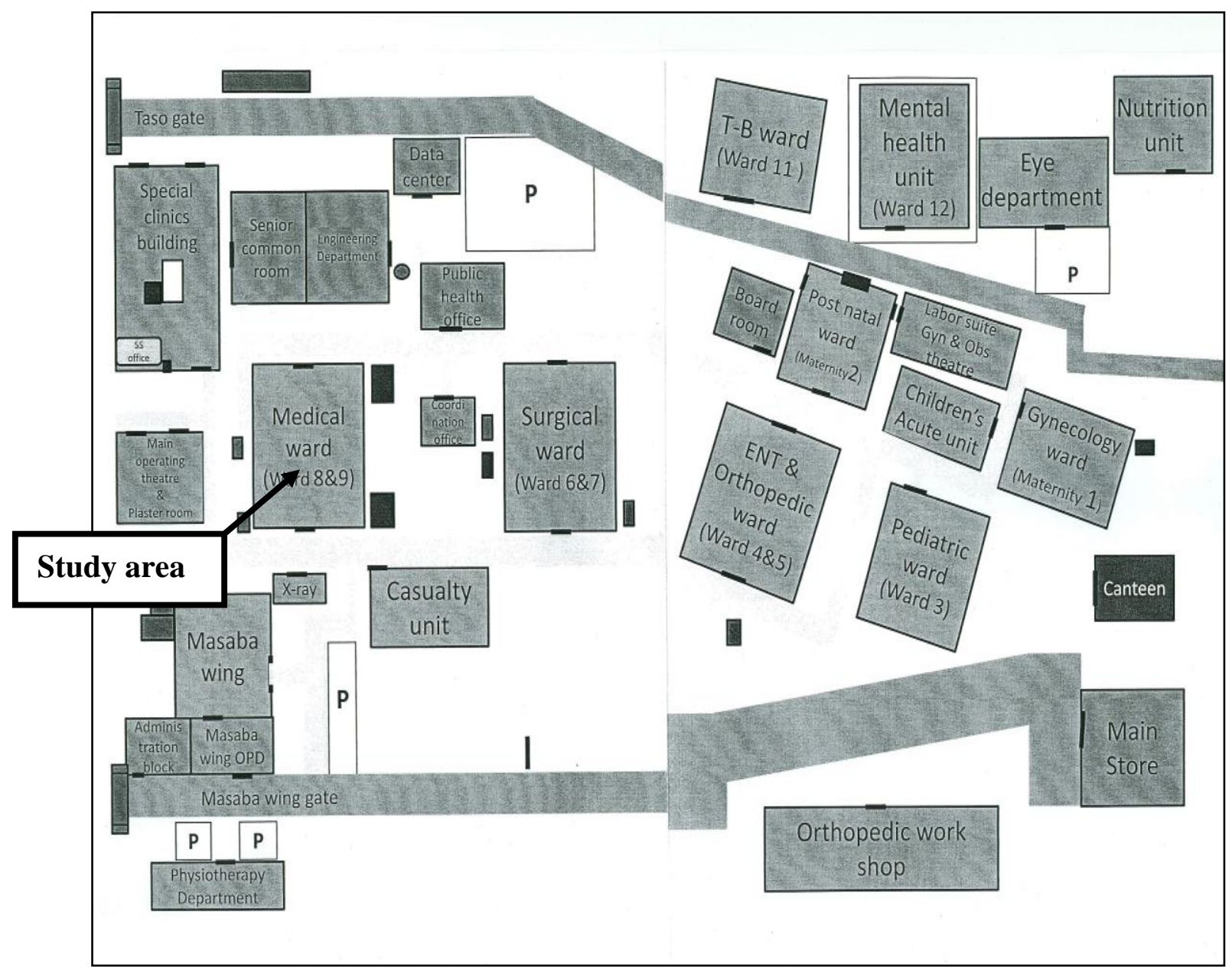

